\begin{abstract}
:
Title: "Modeling and Testing of Docking and Berthing Mechanisms"

The Contact Dynamics Simulation Laboratory (CDSL) of the Marshall Space Flight Center provides for refined hardware-in-the-loop real-time simulation of docking and berthing mechanisms and associated control systems. This facility is employed to verify the performance of docking/berthing mechanisms during Earth-orbit operations, determine the capture envelope of docking/berthing devices, measure contact loads at vehicle interfaces, and evaluate visual cues for man-in-the-loop operations. The CDSL has developed test verified analytical models of such systems as the ISS Common Berthing Mechanism and HST Three Point Docking Mechanism. This paper will describe the modeling and test techniques employed at the CDSL and present results from recent programs.
\end{abstract}

Authors: D. P. Hall, M. M. Slone, NASA Marshall Space Flight Ctr.; P. A. Tobbe, Dynamic Concepts, Inc. 


\title{
Modeling and testing of docking and berthing mechanisms
}

\author{
D. P. Hall ${ }^{\mathrm{a}}$, M. M. Slone ${ }^{\mathrm{a}}$, P. A. Tobbe ${ }^{\mathrm{b}}$ \\ ${ }^{2}$ National Aeronautics and Space Administration, Marshall Space Flight Center, AL, USA 35812; \\ bynamic Concepts, Inc., 6700 Odyssey Drive, Suite 202 \\ Huntsville, AL, USA 35812
}

\begin{abstract}
The Contact Dynamics Simulation Laboratory (CDSL) of the Marshall Space Flight Center provides for refined hardware-in-the-loop real-time simulation of docking and berthing mechanisms and associated control systems. This facility is employed to verify the performance of docking/berthing mechanisms during Earth-orbit operations, determine the capture envelope of docking/berthing devices, measure contact loads at vehicle interfaces, and evaluate visual cues for man-in-the-loop operations. The CDSL has developed test verified analytical models of such systems as the International Space Station (ISS) Common Berthing Mechanism (CBM) and the Hubble Space Telescope (HST) Three Point Docking Mechanism. This paper will describe the modeling and test techniques employed at the CDSL and present results from recent programs.
\end{abstract}

Keywords: Docking, berthing, contact dynamics

\section{INTRODUCTION}

As different space vehicles are developed, interfaces between the vehicles are also developed, creating a need to verify the operation of the interfaces. The Marshall Space Flight Center's Contact-Dynamics Simulation Lab (CDSL) was developed in response to the need to evaluate the Earth-orbit dynamic response of the interfacing mechanisms. The CDSL is a simulation test bed for the study of the contact-dynamics of full-scale docking and berthing mechanisms.

The CDSL allows engineers to simulate how a docking or berthing mechanism would behave in earth orbit under a variety of conditions. This simulation can be used to determine the capture envelope of docking and berthing devices. Through the use of force and torque sensor data recorded during a simulation, the CDSL can also reveal the stresses a device will experience once in space. Past simulations have resulted in the re-design of some mechanisms, improving their performance, and ensuring that they could perform the task for which they were designed.

\section{FACILITY DESCRIPTION}

The CDSL of the Marshall Space Flight Center provides for refined hardware-in-the-loop (HWLL) real-time simulation of docking and berthing mechanisms and associated control systems. The CDSL (Figure 1) is employed to verify the performance of docking/berthing mechanisms during on-orbit operations, determine the capture envelope of docking/berthing devices, measure contact loads at vehicle interfaces, and evaluate visual cues for man-in-the-loop operations. The CDSL serves as a test bed for the study of the contact dynamics of full scale docking and berthing mechanisms by allowing engineers to simulate how a docking or berthing mechanism would perform in orbit under an array of conditions. In 1994 the National Science and Technology Council (NSTC) determined that the CDSL capabilities were "world class and unique." The NSTC defined "world class" as "a facility that provides a quality, capability, capacity, product, technology, condition, or process recognized by the world aerospace community as among the best in the world."

A typical berthing or docking mechanism is composed of two mating components, one for each vehicle. A docking mechanism is a kinetic energy assisted mechanism, while a berthing mechanism is a manipulator assisted mechanism. In the CDSL, one component is attached to a motion-base, while the other component is mounted to a force and torque 
sensor fixed into the support structure above the motion-base. The motion-base is a hydraulically driven CAE-LINK Stewart Platform controlled by a real-time computer system. The computer controlled motion-based simulates the relative motion of the docking mechanisms in six degrees of freedom. The relative motion is based upon any combination of (1) a real-time simulation of multiple orbiting vehicles, (2) real-time simulation of the Shuttle and Space Station Remote Manipulator System (RMS), and (3) a remote man-in-the-loop station with computer-animated or live TV graphics.

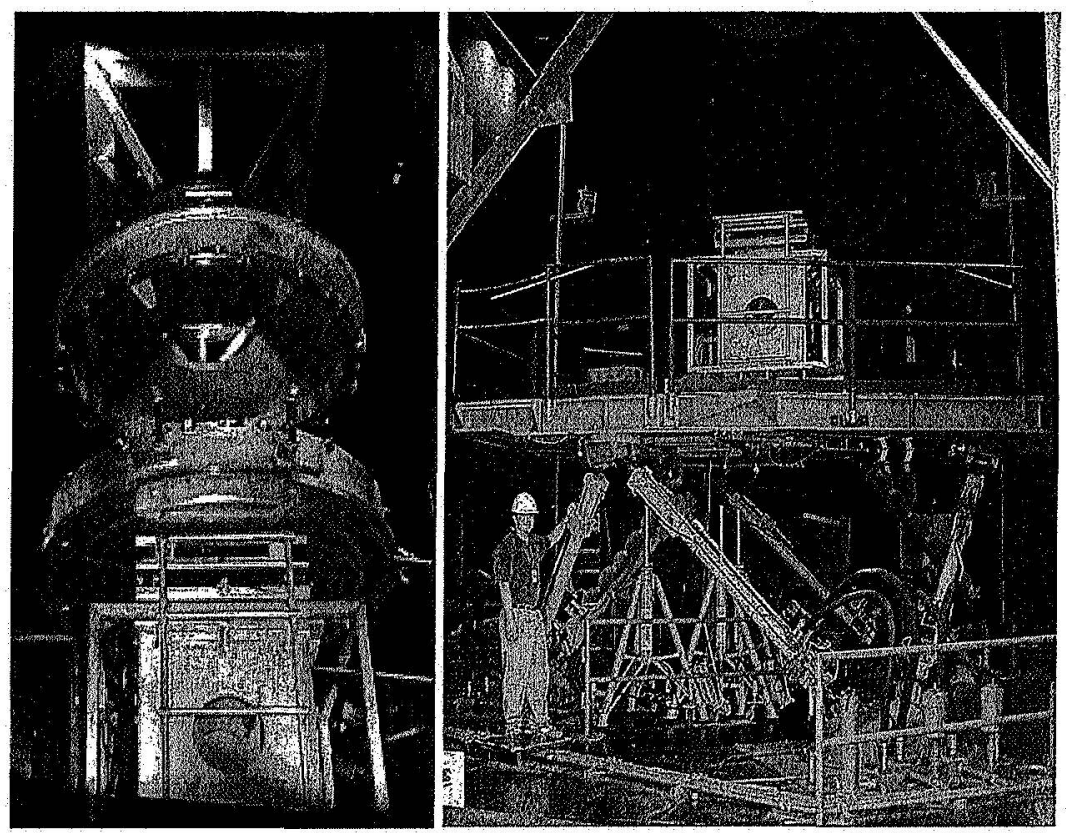

Figure 1: Contact Dynamics Simulation Laboratory

The simulation can be used to dock two distinct vehicles or berth two objects together using one of the RMS models. The two body docking model (Figure 2) simulates the six degree of freedom rigid body motion of each vehicle coupled with flexible body characteristics. The simulation supports interfaces for vehicle guidance, navigation, and control (GN\&C) models, actuator models, environmental models, and measured or analytical contact loads.

The Shuttle Remote Manipulator System (SRMS) simulation is a model of the flexible booms and joints of the SRMS, the joint servos, the SRMS control system, flexible payloads, and base vehicles. The real-time SRMS simulation currently runs in a parallel format and was validated to flight and simulation data. The pilot workstation includes the hand control joysticks, the SRMS control panel graphical user interface, computer-generated and live TV graphics. The SRMS control panel is the user interface to operate the SRMS. The panel is composed of switches that select the mode of operation, joint to be controlled, brakes, and end effector. The panel also displays data such as joint angles, point of resolution (POR) position and orientation, POR velocity, commanded rates, brake status, and mode of operation.

The motion-base is commanded by the real-time computer which hosts the digital models of the docking or berthing environment. When the two components of the berthing mechanism come in contact, the Force-Moment sensors send the contact loads to the computer, which then uses the information to calculate the resulting motion of the vehicles for the docking simulation or arm response for the berthing simulation. The relative motion between the test articles is then computed and leg length commands for the motion-base are generated. The test article is protected through several safety sensors that monitor the loads imparted by the motion-base. The computer also sends data to workstations to drive graphics displays of the simulated vehicles. 
The CDSL has been utilized in the validation of a number of docking and berthing mechanisms over the past decades. A list of several of these mechanisms includes: Apollo / Skylab, Space Station Docking Mechanism (McDonnell Douglass Design), Orbital Maneuvering Vehicle (OMV) Three Point Docking Mechanism, Space Station Freedom / Alpha

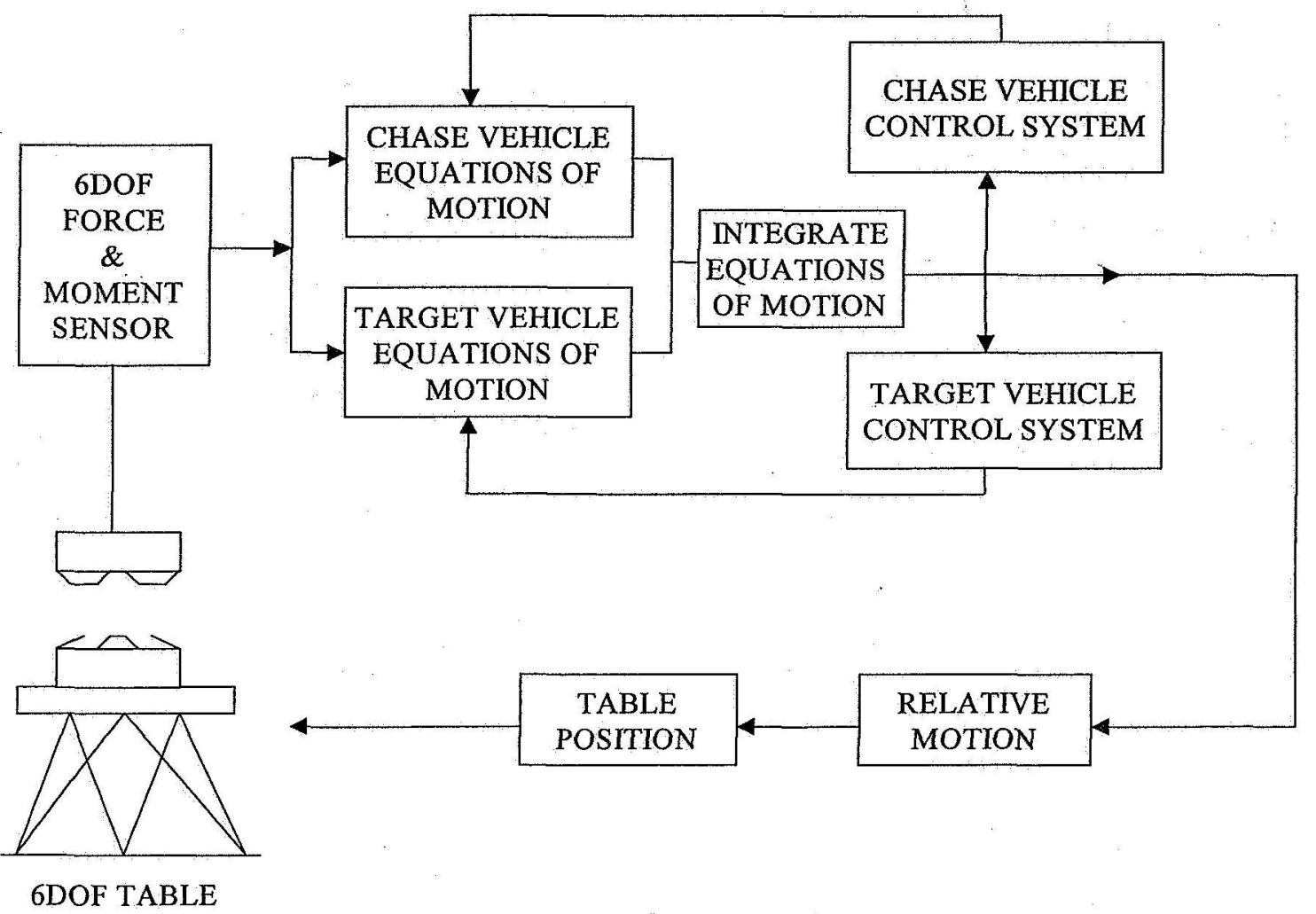

Common Berthing Mechanism, Space Shuttle Remote Manipulator System End Effector, ISS Common Berthing Mechanism, and the Orbital Express Docking Mechanism.

Figure 2: Two body docking model simulation

\section{MECHANISM MODELING}

An analytical mechanism model is an invaluable tool that supports the design, test, verification, integration and operation of the mechanism throughout its design life. Mechanism models; when integrated with digital vehicle and manipulator simulations, can be used to predict vehicle / manipulator interface loads, mechanism component loads, and dynamic capture envelopes. These models can also be used to aid in the design of capture mechanism and alignment surfaces, develop integrated operation procedures with vehicle and manipulator control systems, and define test fixture requirements.

Mechanism models should be developed early in the design cycle of the mating system to support the evaluation of design requirements and system performance. Using prototype hardware and component/system testing, the mechanism models should be upgraded to correlate with test data and evaluate future design improvements. The final configuration controlled model can then be used to support pre-flight analysis, procedure development, training, and flight anomaly resolution.

In models of passive mechanisms with no active control of components, analytical models typically consist of modules which compute the relative motion between contacting surfaces and resultant contact/alignment loads. However, for 
more complex systems, such as the Common Berthing Mechanism (Figure 3), models also include modules for mechanism sensors, actuators, control systems, and capture system dynamics.

Contact force models are traditionally based on the method of soft constraints or penalty method. In this technique, mechanism contact surfaces are approximated either through simple geometric equations (i.e. lines, planes, arcs, etc) or numerically through splines and polygons. Contact is determined by checking for intersection or penetration between the corresponding surfaces and calculating mutually perpendicular forces proportional to the depth of penetration.

The CBM is the berthing mechanism that structurally connects components of the International Space Station. The CBM mechanism model consists of ring to ring, guide to ring, pin to socket, plunger to strike plate, duckhead bumper to follower, and capture latch tip to fitting contact force models. A dynamic model of the capture latches (four bar linkage equations of motion) was developed and driven by motor shaft drive torques and latch tip contact forces. The closed loop latch control system, drive train, motor, and drive arm motion sensors was also simulated and interfaced to the CBM operator and capture latch dynamics. The net interface loads between the halves of the CBM mechanism were output to a digital berthing simulation of the Space Shuttle, ISS, and RMS.

The CDSL was used exclusively throughout the 1990's in support of the CBM development and qualification test programs. In conjunction with a detailed mechanism modeling effort, CDSL tests validated CBM performance requirements through the accurate determination of the capture envelope and Space Shuttle and Space Station RMS interface loads. The CDSL also played a major role in the development and testing of coordinated RMS and CBM operational procedures for nominal and off nominal berthing scenarios. This effort culminated in a validated CBM test bed and test verified mechanism model used for crew training and mission support.



Figure 3: Common Berthing Mechanism

\section{HUBBLE SPACE TELESCOPE ANALYSIS AND TEST PROGRAM}


In the summer of 2005, a test of the soft capture mechanism (SCM) prototype hardware in support of the Hubble Robotic Servicing and Deorbit Mission (HRSDM) was performed in the CDSL. The objective of the CDSL testing was to characterize docking and berthing envelopes in terms of physical offsets and rates (translational and angular) allowable for successful docking and berthing including consideration of allowable HST loads. In particular, the tests sought to verify the adequacy of GN\&C requirements on spacecraft positional, angular, and rate accuracy during direct docking and berthing. An engineering evaluation will be performed of berthing the HST to the new propulsion module during the Shuttle-based Servicing Mission, including contact dynamics and carrier-mounted cameras to support the berthing sequence.

The following compliance matrix (Table 1) illustrates how the Test Objectives were to be achieved during the tests.

\begin{tabular}{|c|c|}
\hline 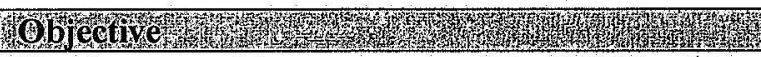 & rompliance eriterat \\
\hline $\begin{array}{l}\text { Verify successful engagement of the HST berthing } \\
\text { pins to SCM during direct docking in the presence } \\
\text { of 3-sigma positional, angular, and rate errors. }\end{array}$ & Engagement of all berthing pins with the SCM. \\
\hline $\begin{array}{l}\text { Quantify loads on HST and Hubble Rescue Vehicle } \\
\text { (HRV) during direct docking in the presence of 3- } \\
\text { sigma errors. }\end{array}$ & $\begin{array}{l}\text { Time history of loads on both vehicles correlated } \\
\text { with positions of each berthing pin with respect to } \\
\text { its respective SCM. }\end{array}$ \\
\hline $\begin{array}{l}\text { Verify successful engagement of the HST berthing } \\
\text { pins to SCM during berthing in the presence of 3- } \\
\text { sigma positional, angular, and rate errors. }\end{array}$ & $\begin{array}{l}\text { Simultaneous engagement of all berthing pins with } \\
\text { the SCM. }\end{array}$ \\
\hline $\begin{array}{l}\text { Quantify loads on HST and HRV during berthing in } \\
\text { the presence of 3-sigma errors. }\end{array}$ & $\begin{array}{l}\text { Time history of loads on both vehicles correlated } \\
\text { with positions of each berthing pin with respect to } \\
\text { its respective SCM. }\end{array}$ \\
\hline $\begin{array}{l}\text { Evaluate potential modifications to the positional, } \\
\text { angular, and rate requirements for both direct } \\
\text { docking and berthing. }\end{array}$ & $\begin{array}{l}\text { Recommendations on required contractions or } \\
\text { recommended expansions of the required relative } \\
\text { vehicle control errors during direct docking and } \\
\text { berthing. Recommended alternate sets of relative } \\
\text { vehicle control errors are verified as resulting in a } \\
\text { successful SCM engagement. }\end{array}$ \\
\hline $\begin{array}{l}\text { Develop a validated contact dynamics model of a } \\
\text { single SCM and of the three-SCM system. }\end{array}$ & $\begin{array}{l}\text { A validated single-SCM and a validated three-SCM } \\
\text { model are delivered to GSFC, and are verified to be } \\
\text { compatible with existing modeling systems. }\end{array}$ \\
\hline $\begin{array}{l}\text { Evaluate camera positions for HST SM } 4 \text { carriers to } \\
\text { support berthing of HST in the Space Shuttle. }\end{array}$ & $\begin{array}{l}\text { Establish multiple camera locations and associated } \\
\text { field of views to evaluate adequacy of system. }\end{array}$ \\
\hline
\end{tabular}

Table 1: Hubble Robotic Servicing and Deorbit Mission Soft Capture Mechanism Test Compliance Matrix

\subsection{HST SCM modeling}

In support of the HWIL testing and post test analyses, an analytical mechanism model of the SCM (Figure 4) was developed. Initial mechanism model requirements were derived from the test objectives. The initial SCM model was integrated with the two body simulation and used to determine CDSL component test requirements and run cases. Results from the test data were correlated with the model to produce a test verified model for integration into digital docking and berthing simulations.

The HRV contacting surfaces included six guide to berthing pin pairs (Figures 4 and 5), three guide extension to berthing pin pairs (Figure 5), six saddle to berthing pin pairs (Figure 6), and twelve SCM soft latch finger to berthing pin pairs (Figure 7). Contact existed for any and all contact pairs at a given time. The individual contact forces and moments were accumulated before outputting to the 2-body simulation. In addition, the guides, saddles, and fingers were all capable of motion. 
The guide contacting surface (Figure 5) was modeled as two arcs and eight line segments, while the berthing pin (Figure 4) was modeled as a single line segment with a radius to define its cylindrical nature. There were three guide structures (each with a front and back side) that only differ in location and orientation with respect to the global active frame. There were also three berthing pins, each of which could have contacted with one guide. In addition, there were three guide extensions, each made up of two lines that extended to the left and right of each guide. The purpose of these contact pairs was to stop the simulation if a berthing pin moved beyond the nominal capture envelope. Effort has been made to only include the basic geometry parameters necessary to define a given contact surface. The contact force model (CFM) code will loop through all possible contacts between the berthing pin line and the guide arcs and lines, then find the minimum distance between the two bodies. Contact forces and moments are computed if penetration exists.

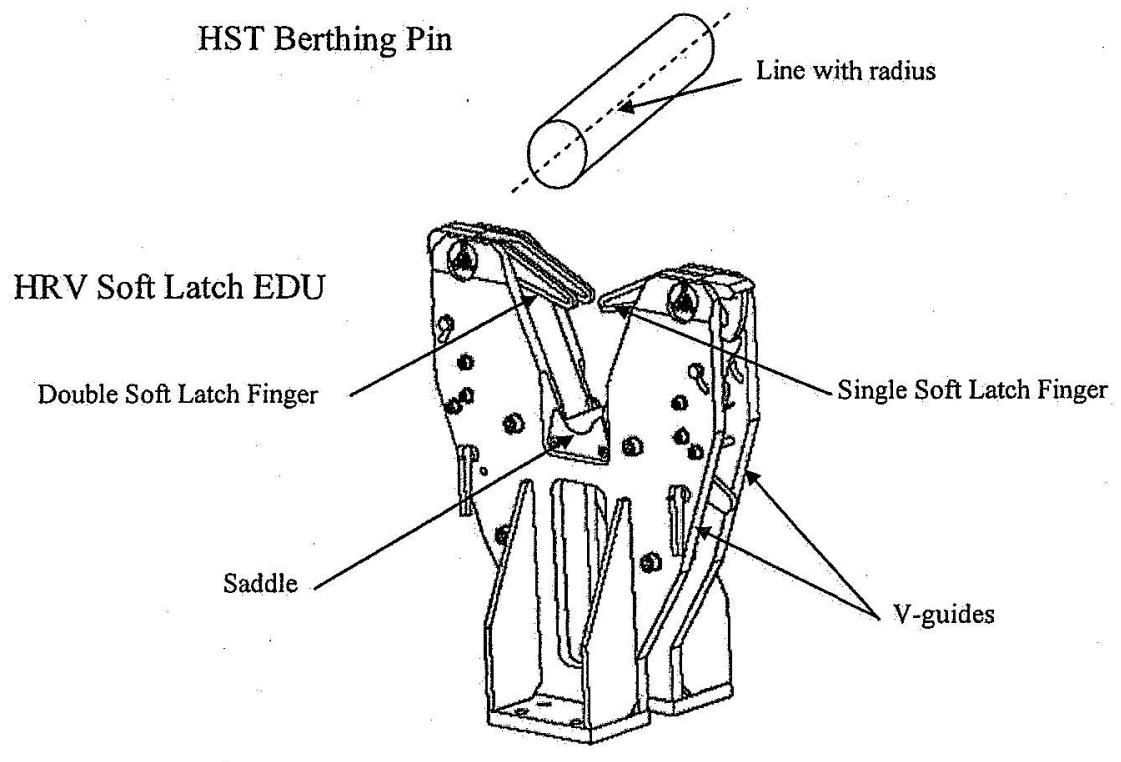

Figure 4: HRV Soft Latch and HST Berthing Pin

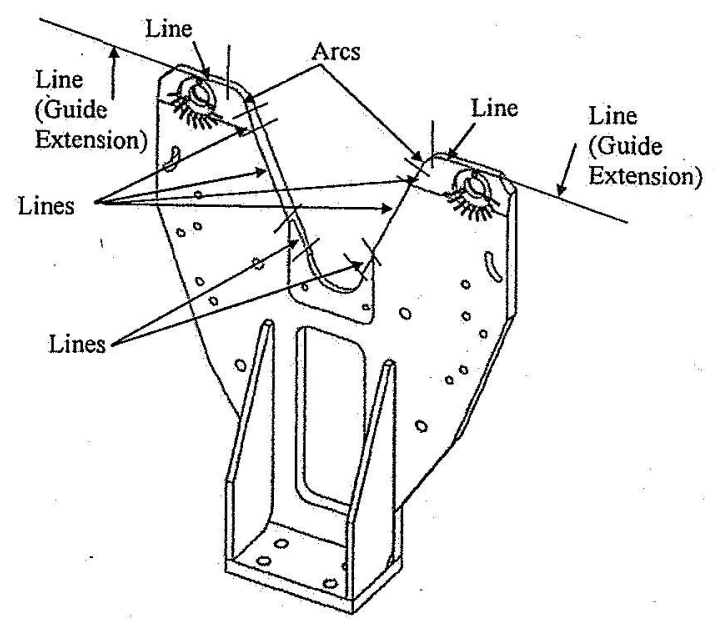

Figure 5: HRV Soft Latch and HST Berthing Pin 
As shown in Figure 6, the saddle is made up of two lines and one arc. Each of the three saddles also had a front and back side.

The SCM single soft latch finger and double soft latch finger (Figure 7) was also modeled. Each finger consisted of 5 lines and $1 \mathrm{arc}$, with the orientation and the separation of the front and back sides being the only difference between the single and double finger geometries. The CFM code looped through the berthing pin line contacting the edges of the fingers, each berthing pin could have contacted one set of double and single latches. The minimum distance between the contacting surfaces of the berthing pin and fingers was deduced, with contact forces and moments calculated if applicable.

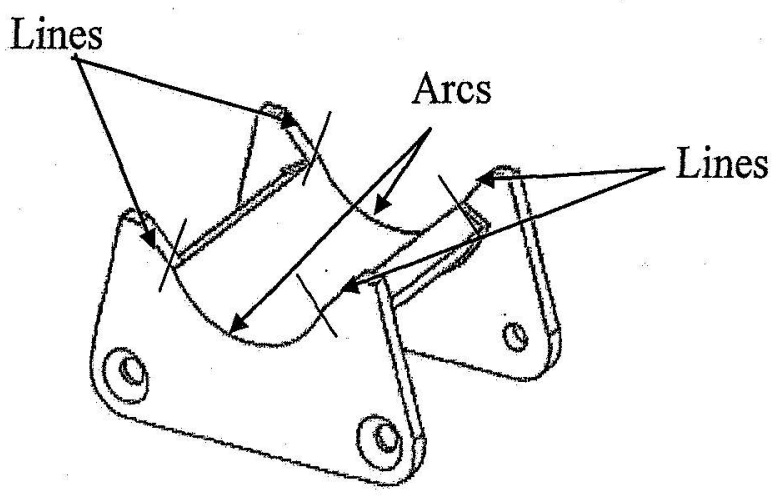

Figure 6: HRV Saddle

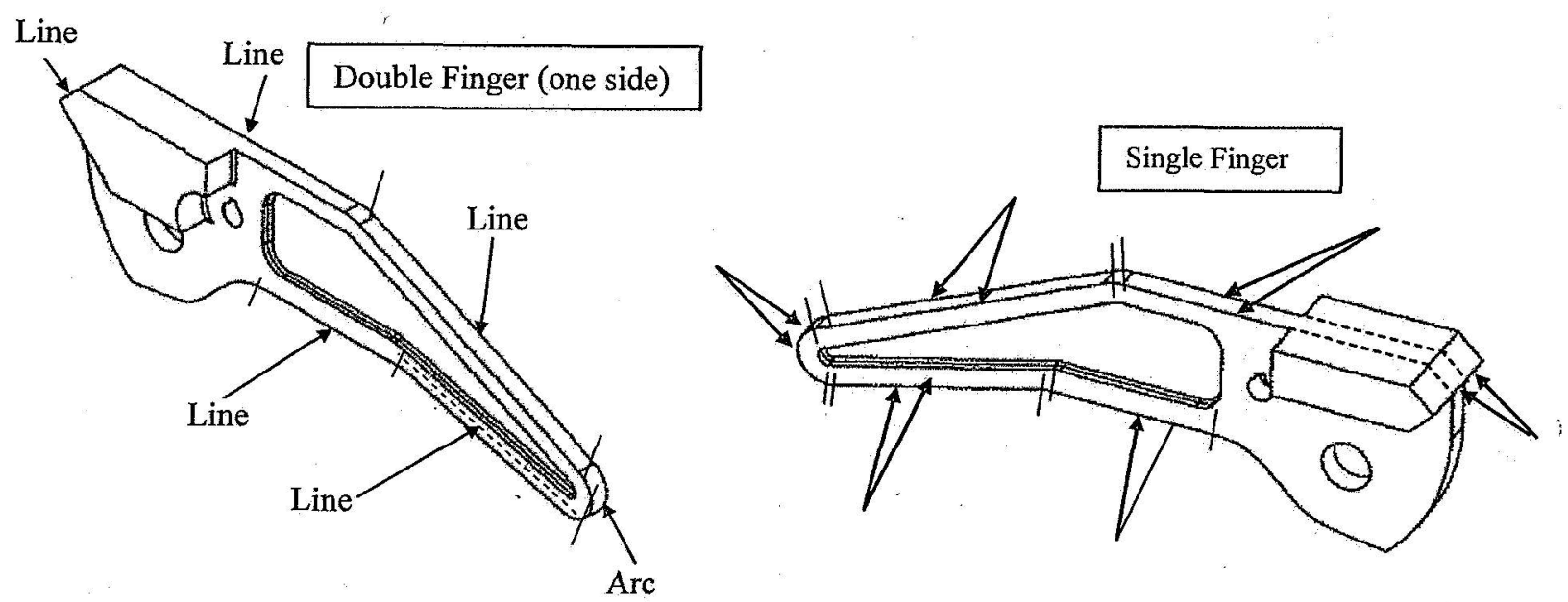

Figure 7: HRV SCM Double Finger (one side) and HRV SCM Single Finger

\subsection{Monte Carlo runs to support requirements and test}

A Monte Carlo analysis was performed with the initial mechanism model and two body simulation to develop valid run cases for HWIL testing and model correlation. The approach consisted of generating the random initial conditions (ICs) for the test cases, statistically reducing the non-contact initial conditions, and generating the appropriate input files for the simulation. Source code was written in Matlab to perform the randomization and reduction. 
Table 2 contains the maximum lateral offsets in y and $z$, and roll, pitch, and yaw angular. The axial separation was kept at 5.52 inches for all cases, which allowed the bottom of the HST berthing pin to be at the same level as the top of the capture latch guides without additional angular offsets. For this set of runs, a matrix of 50,000 x 5 uniformly distributed random numbers was generated. Each random number, with a range of -1 to 1 , was then multiplied by the appropriate maximum value to obtain a uniform distribution within each variable's maximum range.

\begin{tabular}{|l|l|}
\hline Offset & Limit \\
\hline $\mathrm{y}$ & $\pm 2.7 \mathrm{in}$ \\
\hline $\mathrm{z}$ & $\pm 3.1 \mathrm{in}$ \\
\hline roll & $\pm 4.2^{\circ}$ \\
\hline pitch & $\pm 2.519^{\circ}$ \\
\hline yaw & $\pm 2.179^{\circ}$ \\
\hline
\end{tabular}

Table 2: Maximum Position Offsets

The resulting inputs files were screened for initial conditions which were in contact. Of the original 50,000 random IC cases, there were 17,067 cases that did not have contact and did not extend over the upper, flat portion of the guides. This reduced set was then used to find the standard deviation of each variable. The data was normalized by the standard deviation, and the ceiling of the absolute value was taken to get the positive integer sigma values for each variable. The normalized data was then iterated through and only unique combinations of sigma values were stored in an ultrareduced data set. A total of 80 statistically unique cases were identified using this method.

The remaining initial conditions were combined with 9 sets of relative velocities to form 720 cases run in the Monte Carlo simulation. In order to simulate a run that can be made in hardware, the random ICs must be backed up to a safe initial position for the hardware. To accomplish this, a negative integration was performed from the original IC to a point where each berthing pin was at least 6.02 inches from the center of each guide's saddle. During the negative integration, SCM soft capture latch finger contact forces were turned off so that the movement was unobstructed. However, all other contact forces were active and a run was terminated if there was contact with the guide.

During positive integration, a run was stopped if there was contact with an extended section of the guide, indicating that the berthing pin was headed either to the extreme left or right of the latch guides. Runs were also terminated if the axial separation was too large, indicating that the HST berthing pins have bounced off the guides and are headed in the wrong direction.

The simulation results indicated that there were 220 (31\%) full captures (each latch captured a berthing pin), 247 (34\%) partial captures (one or two latches captured a pin), and 253 (35\%) cases with no capture. There was a low success rate for capture in part because combinations of high sigma values were treated the same as those with low sigma values. A number of cases tested the outer limits of the capture envelope, where velocities became essential in determining whether capture would occur. No inspection of velocity and position was performed; therefore obviously "bad" combinations were allowed to run.

Many of the runs were examined graphically to help explain why capture failed. In most unsuccessful cases the berthing pin hit the top corner of the guide and bounced off, which illustrated the effect of including larger dispersions. Velocity was also very important to a successful capture. Many of the low sigma cases that were easily captured with the nominal and high V1 velocity were not fully captured at the lower velocity. The lower velocity allowed more finger interaction in some cases, or a different angle from which to bounce off the insides of the guides.

\subsection{Facility component requirements definition}


The output of the Monte Carlo runs were screened for maximum loads, displacements, and velocities to determine requirements for the facility force / moment sensors, safety systems, and test article instrumentation. Digital load cells based on strain gages were selected from JR3. Test cases were broken into a low and high velocity groups. Low range / high accuracy force / moment sensors were specified for the low velocity cases to determine minimum approach velocities for capture. High range / less accurate sensors were selected for the high velocity cases. Measured force and moment limits were defined to protect the test article and load sensors. These loads were also used to select the size of facility shear pins and tensile links in the load path of the structure. Encoders were selected for the SCM's soft latch fingers to record the resulting motion of the fingers as they interacted with the berthing pins.

\subsection{Test run matrix definition}

CDSL motion system commands were generated from the simulated relative motion to remove cases outside the limits of the hydraulic table. Test cases were also defined to isolate specific types of contact to support correlation with the analytical mechanism model. One hundred and three HRV / HST docking cases, thirty-eight Shuttle RMS berthing cases, and twenty-four single latch run cases were finalized for testing in the CDSL. Test plans and procedures were written and reviewed. Hardware/software integration activities were then completed before test conduct.

\subsection{Hardware and software integration and checkout}

Mechanical, electrical hardware, and software integration was necessary to mount, control, and monitor the SCM test articles during the simulation tests. The mechanical integration included the HRSDM Soft Capture Mechanism, the HST berthing pin, and the JR3 force-moment sensors. The electrical hardware and software interfaces included the forcemoment sensors, the force-moment sensor receivers, the HRSDM Soft Capture Mechanism (SCM) Finger Encoders, and the SCM Finger Release/Engagement Actuators.

An exploded view of the SCM, HST pin, and the mechanical interfaces to the simulation facility can be seen in Figure 8. The SCM was mounted directly to the Active Interface Plate (AIP). The HST pin was mounted to the Passive Interface Plate (PIP) through the force-moment sensor assembly. The force-moment sensor assembly included the Lower Adapter Plate (LAP), the force-moment sensor, and the Upper Adapter Plate (UAP).

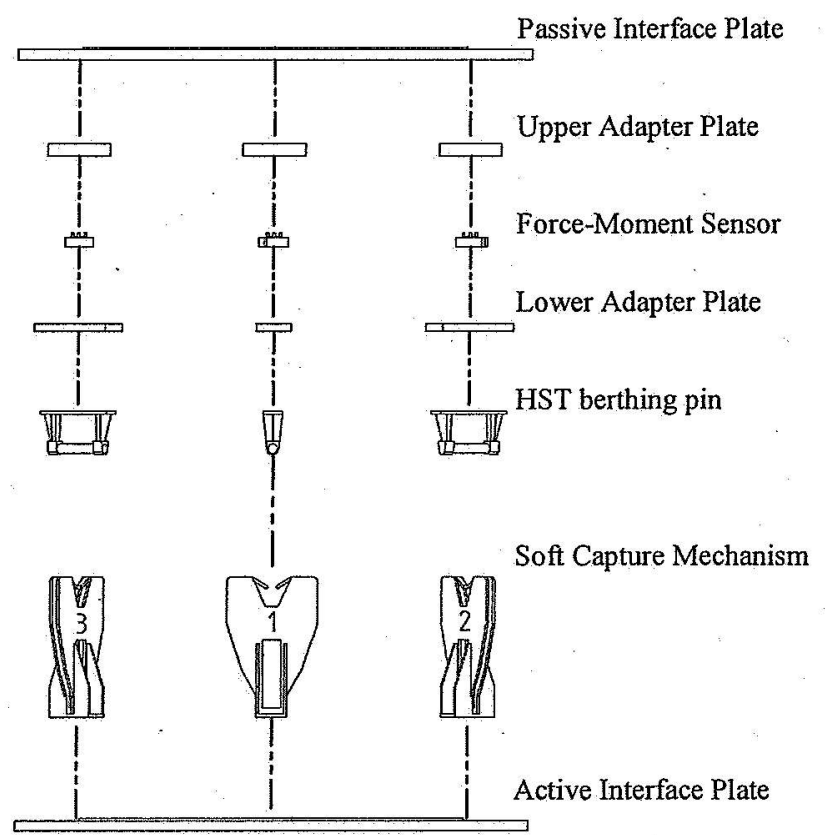

Figure 8: Soft capture mechanisms, HST pins, and facility interfaces 
The integrated CDSL facility and SCM test articles are shown in Figure 9.

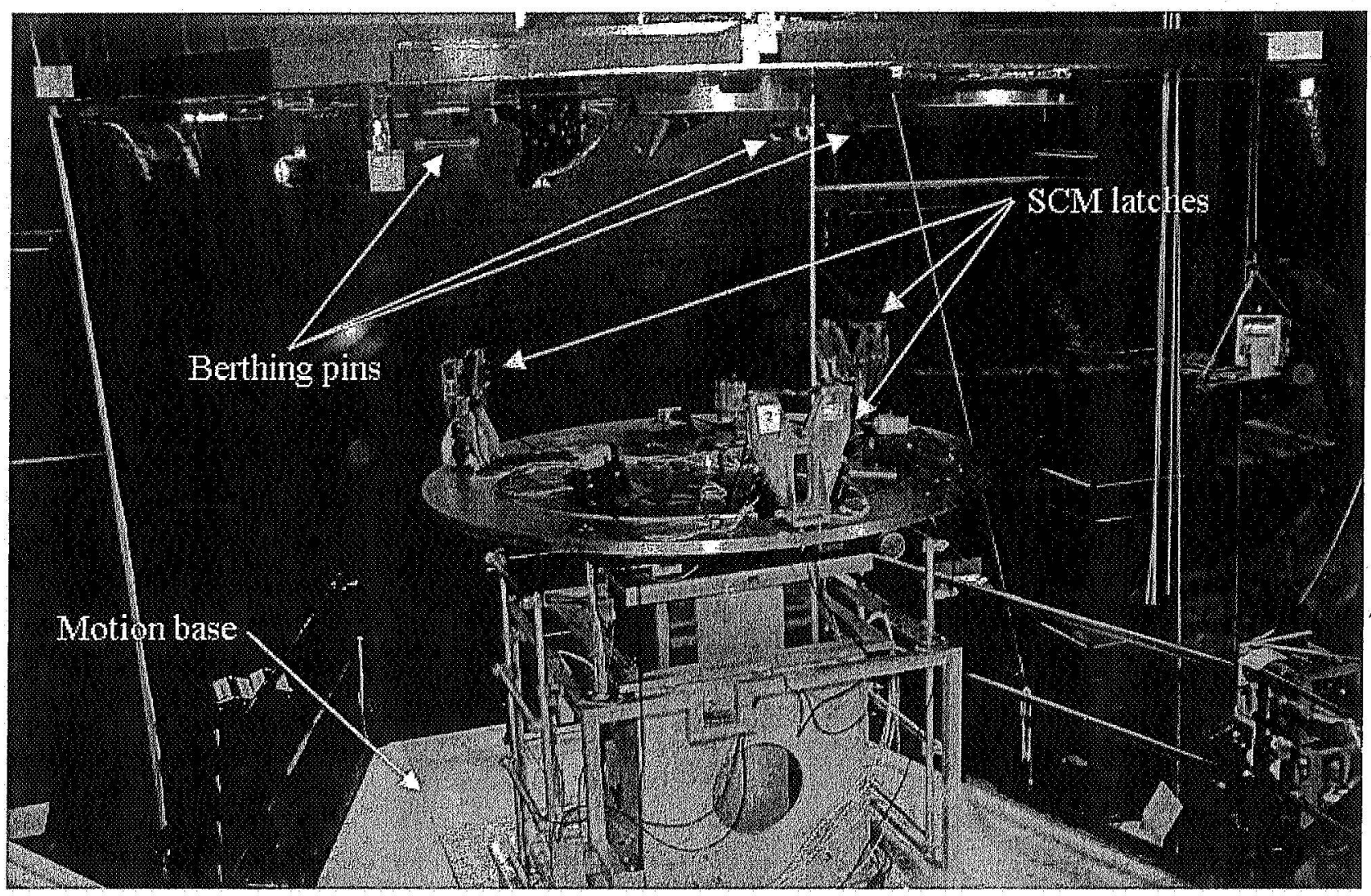

Figure 9: Integrated CDSL facility and SCM test articles

The force-moment sensor electrical integration was accomplished using JR3's DSP-based Force Sensor Receivers (Figure 10) and associated cabling.

The interface for the SCM finger encoders (Figure 11), which were used to determine the angular position of each SCM finger, consisted of a power supply to provide the required power, a VMEbus-based quadrature decoder, and the associated cabling. The VMEbus-based quadrature decoder used was the IP484 Counter Timer Module made by Acromag.
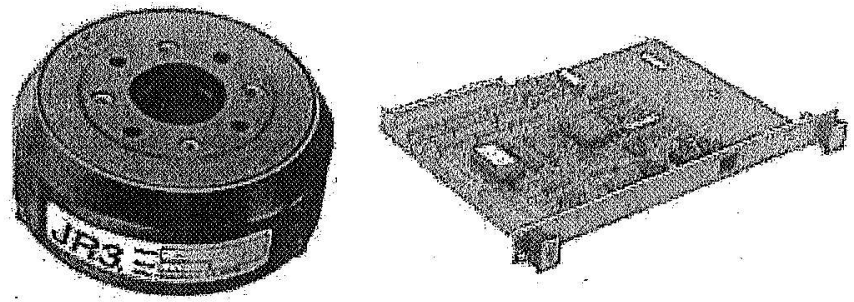

Figure 10: JR3 force-moment sensor and receiver
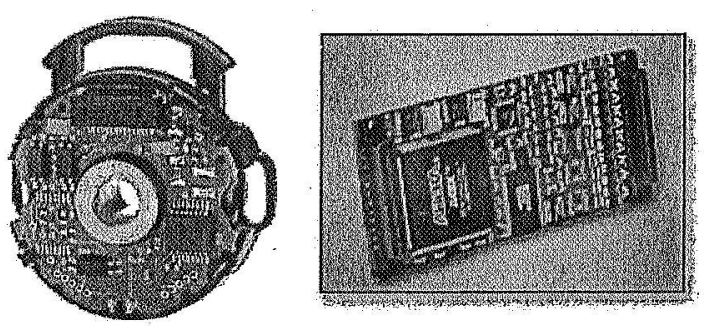

Figure 11: SCM soft latch finger encoder and decoder 
The control for the release/engagement actuators was accomplished using a bank of power relays to energize the extend and retract inputs to the release/engagement actuators. These power relays were controlled using a VMIC VMI-VME2232 relay card.

During test fixture assembly the force-moment sensors were checked to verify accuracy. The simulation software used each sensor's unique, full-scale value to compute the final force and moment values. To verify sensor operation each sensor's tare weight was read and compared to the known load of an HST pin and associated LAP. Next, a ten-pound weight was attached to each HST pin and the resulting $Z$ force checked for sign and magnitude. The orientation of each sensor was checked with $\mathrm{X}$ and $\mathrm{Y}$ forces manually applied, and the resulting moments were checked for magnitude and sign.

Sensor noise was evaluated by observing the sensor's raw output with no force applied to the HST pin. The noise sources consisted of hydraulic pump vibration and electrical system noise. The sensor's raw values were evaluated while the motion system was active. Sensor dead-band values were included in the simulation software to ensure the sensor noise did not impact test results.

The SCM Encoder's power and operation were independently verified before being incorporated into the simulation.

The SCM Release/Engagement Actuators power and operation were independently verified before being incorporated into the simulation.

\subsection{Data reduction and mechanism model correlation}

A series of facility component models which describe the facility motion system, support structure, and force/moment sensor were developed. The facility models account for the influence of the facility components on the simulated environment during HWIL tests. These facility models were used in conjunction with the initial mechanism models and HWIL test data to produce the test correlated mechanism models. The set of mechanism model parameters that were varied to match test data is shown below in Table 3. In addition, table effects were added to the simulation shown in Figure 12 for correlation runs in order to isolate CFM parameters from facility influences. The motion system model modifies the desired or commanded simulation motion through a measured transfer function of the hydraulic table. The resulting motion of the test article is then used to drive the mechanism model. Other facility effects included accuracy and filters in the force / moment sensors and structural resonances in the test fixtures.

\begin{tabular}{|l|l|}
\hline Parameter & Value \\
\hline Damping & Off \\
\hline Stiffness exponent & 1.5 \\
\hline Stiffness & $40,000 \mathrm{lbf} / \mathrm{ft}$ \\
\hline Latch lateral stiffness & $2750 \mathrm{ft}-\mathrm{lbf} / \mathrm{rad}$ \\
\hline Latch axial stiffness & $107,000 \mathrm{lbf} / \mathrm{ft}$ \\
\hline Friction coefficient & 0.15 \\
\hline
\end{tabular}

Table 3: HRV CFM tuning parameters 


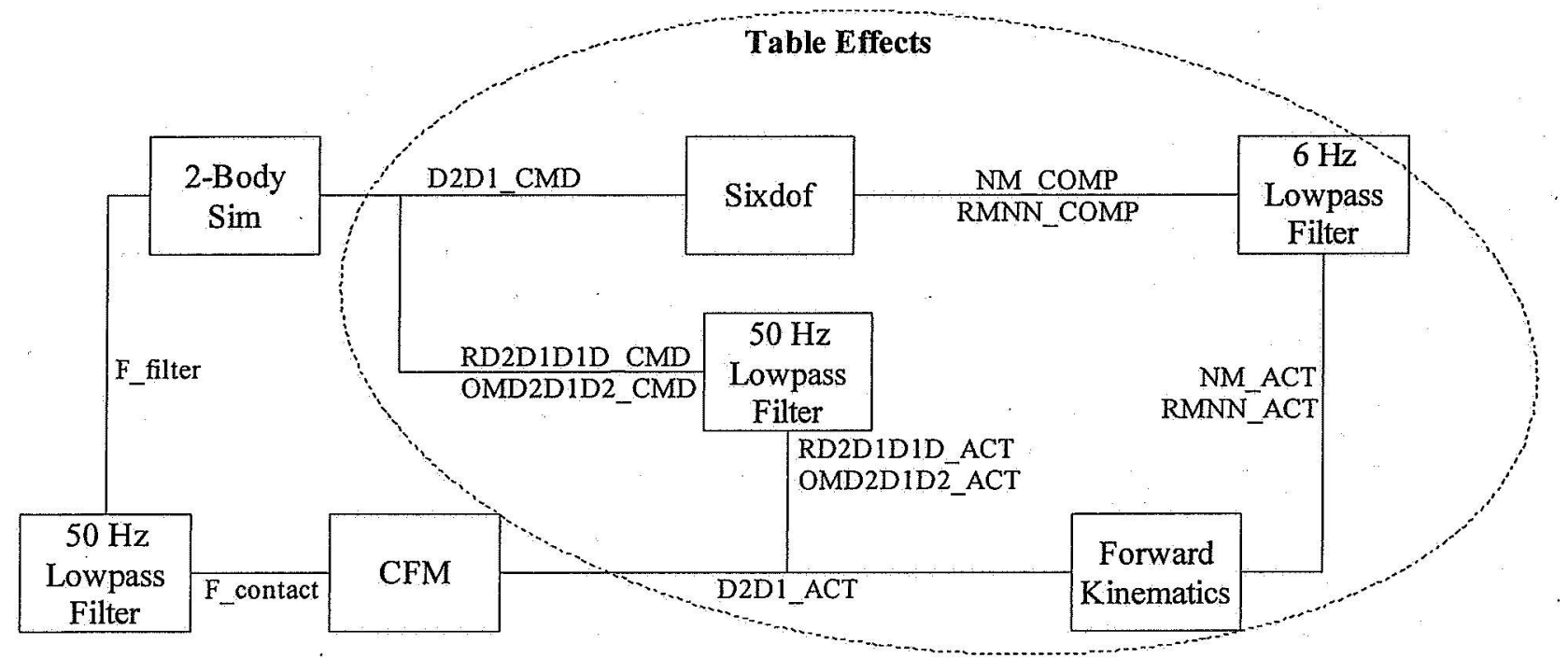

Figure 12: Table Effects Added to Simulation

The variables tuned in the contact model included damping, stiffness exponent, stiffness, friction coefficient, latch axial stiffness, and latch lateral stiffness. The decision to turn damping off was made after observing many runs where the results matched best with little or no damping applied to each geometry element. The contact stiffness exponent, which is used as a way to smooth the contact force as the contact element penetration occurs, was also added to improve correlation with test data.

\subsection{Summary and recommendations}

The Marshall Space Flight Center's (MSFC) CDSL has successfully supported the verification of numerous docking and berthing mechanisms through proven test and analysis techniques. HWIL test and analytical mechanism model requirements, on the component and system levels, were derived from mechanism verification requirements. In addition to supporting the early evaluation of mechanism performance, initial analytical models were used to define test cases and test hardware / software specifications. Test data was used to tune the analytical models for additional support in pre-flight analysis, procedure development, training, and flight anomaly resolution.

The CDSL is currently being upgraded to a Linux-based math model and facility host computer systems. The TRICK simulation environment will be used for real time and non-real time simulations of vehicles, manipulators, and mechanisms. Additional facility upgrades include a family of force / moment sensors with a common interface, redesign of the test article hardware interface for a larger range of mechanism footprints, and improved motion system performance.

\section{ACKNOWLEDGEMENTS}

The authors wish to thank the following persons for their support in this effort: Ms. Linda Brewster, Deputy Branch Chief, Avionics Systems Test Branch, NASA/MSFC; David Hood, Chandra Program Office, NASA/MSFC; the Hubble Robotic Servicing and Deorbit Mission team, NASA/Goddard Space Flight Center (GSFC); and Ashley Hill, Jessica Kessler, Heath Wilson, Tony Williams, Brian Allen, Geri Tobbe, and Chris Cagle-Brown of Dynamic Concepts, Inc. 


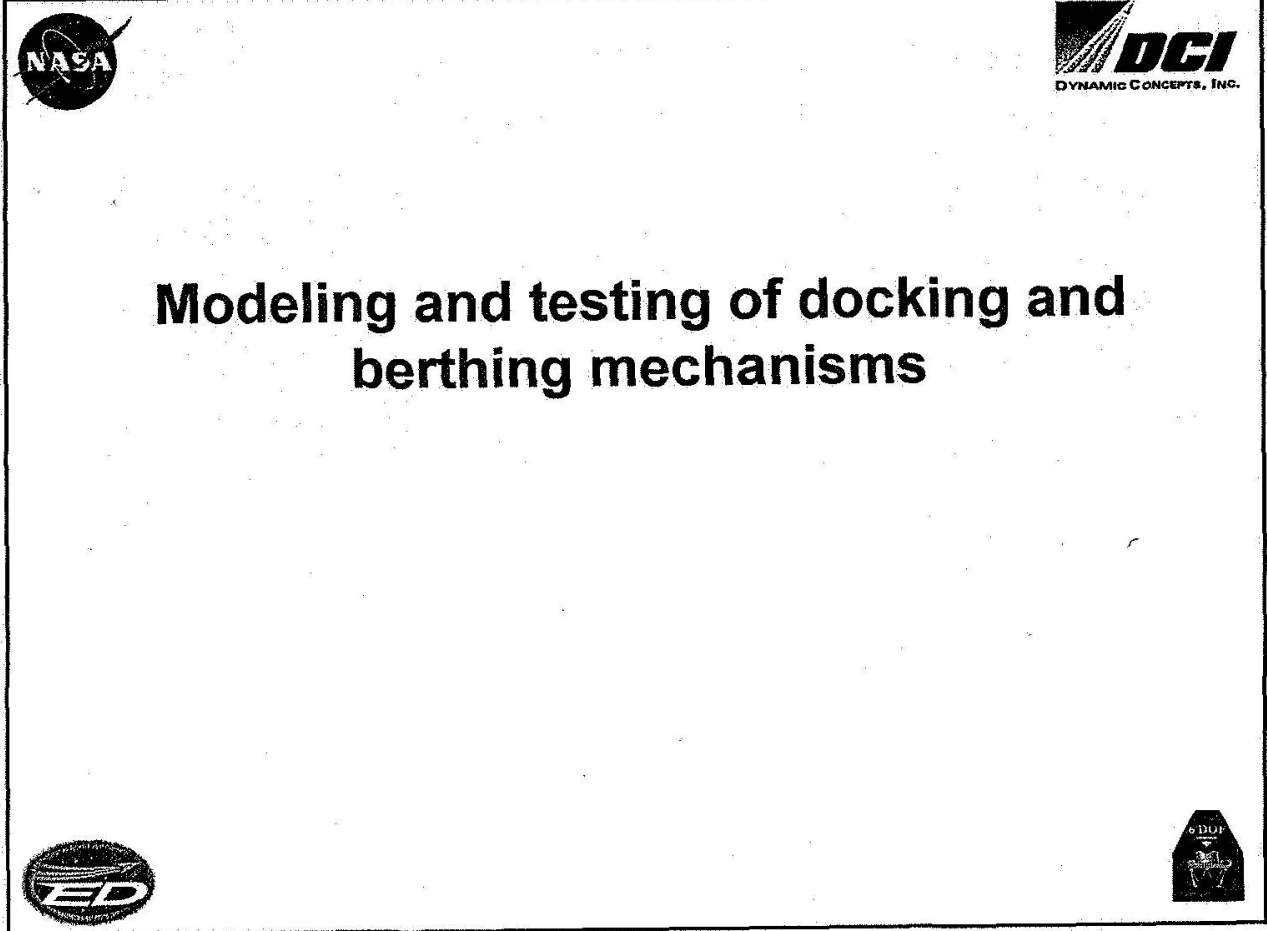

Modeling and testing of docking and berthing mechanisms

Facility Overview

Modeling and Simulation

Examples 

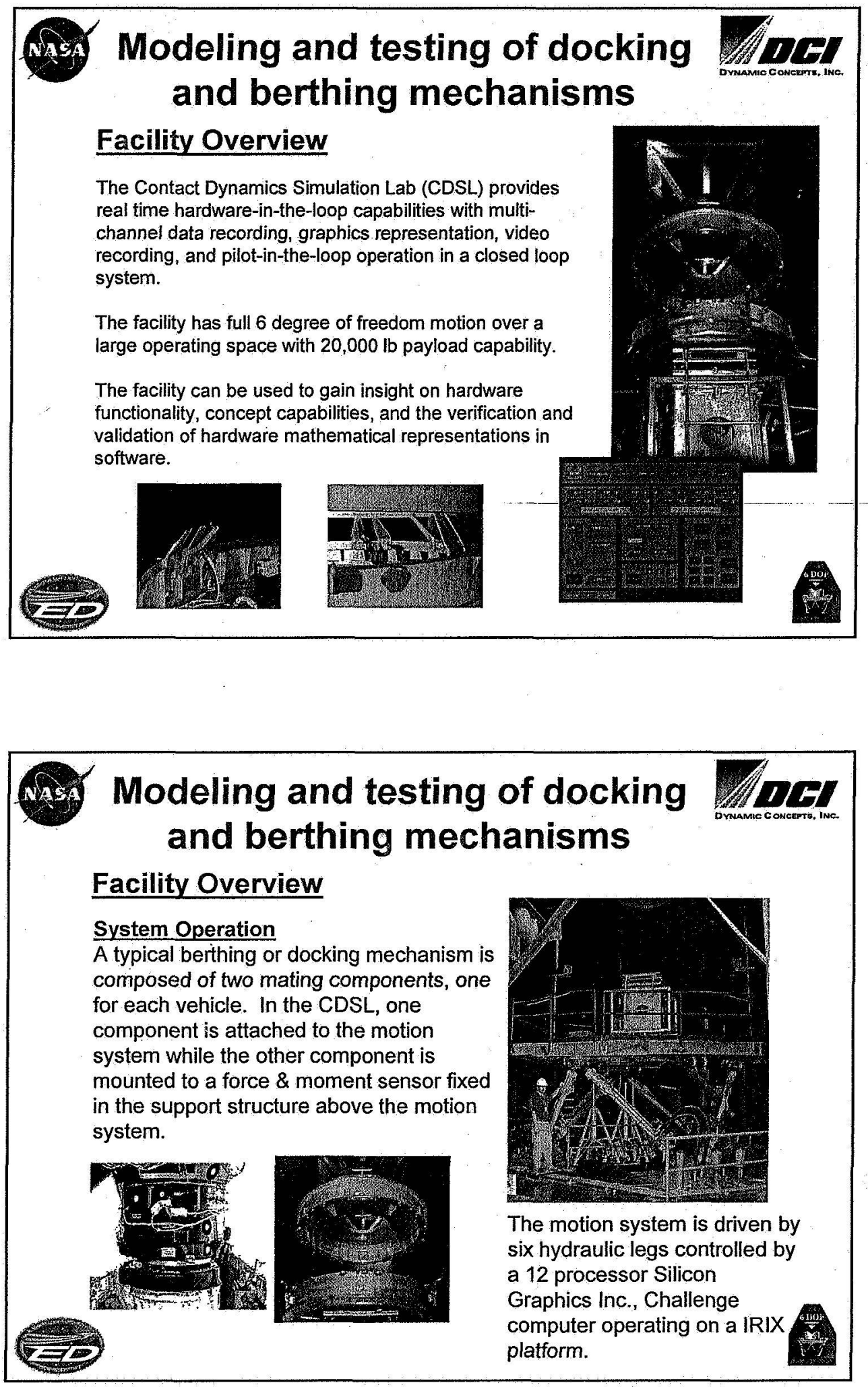

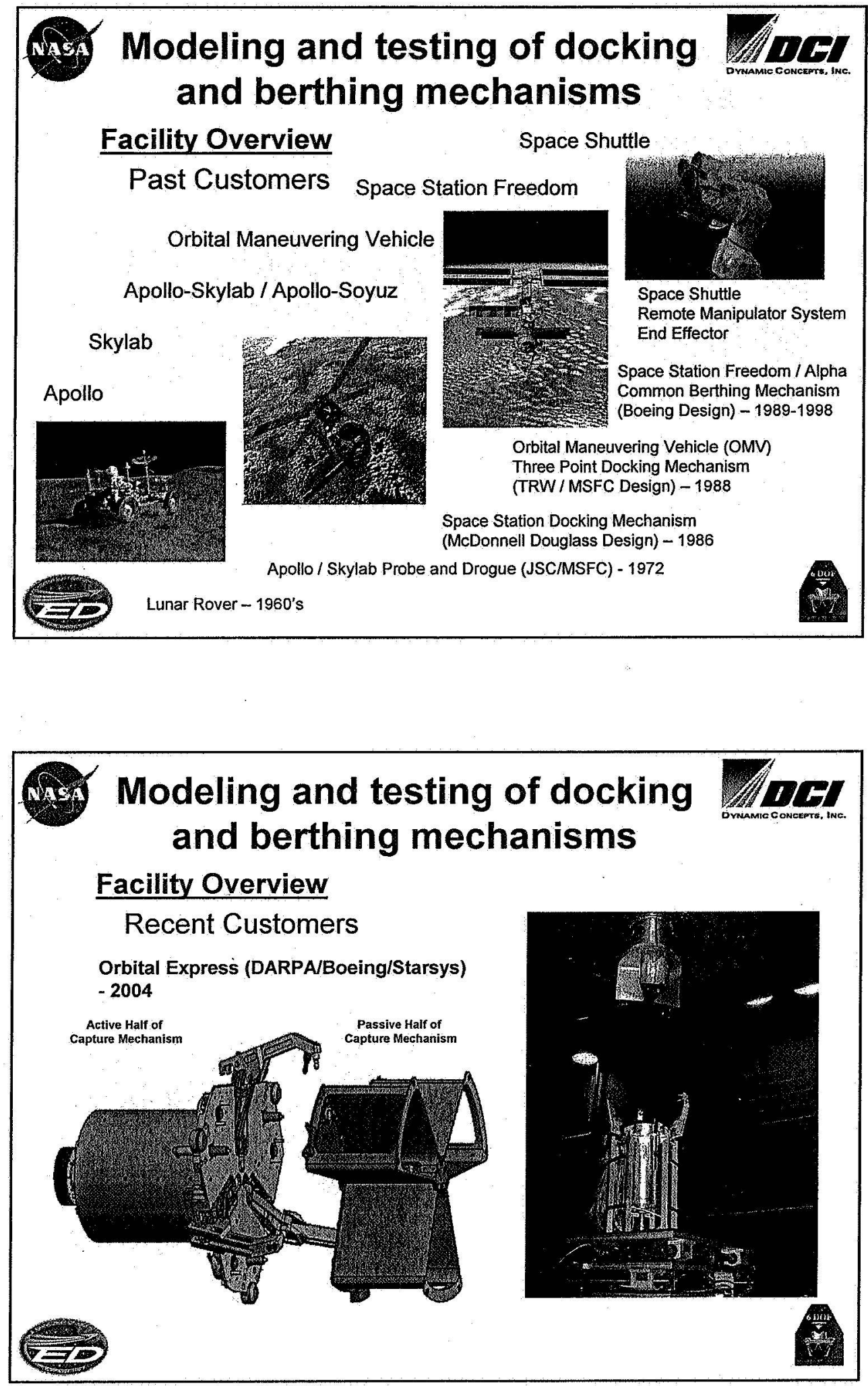


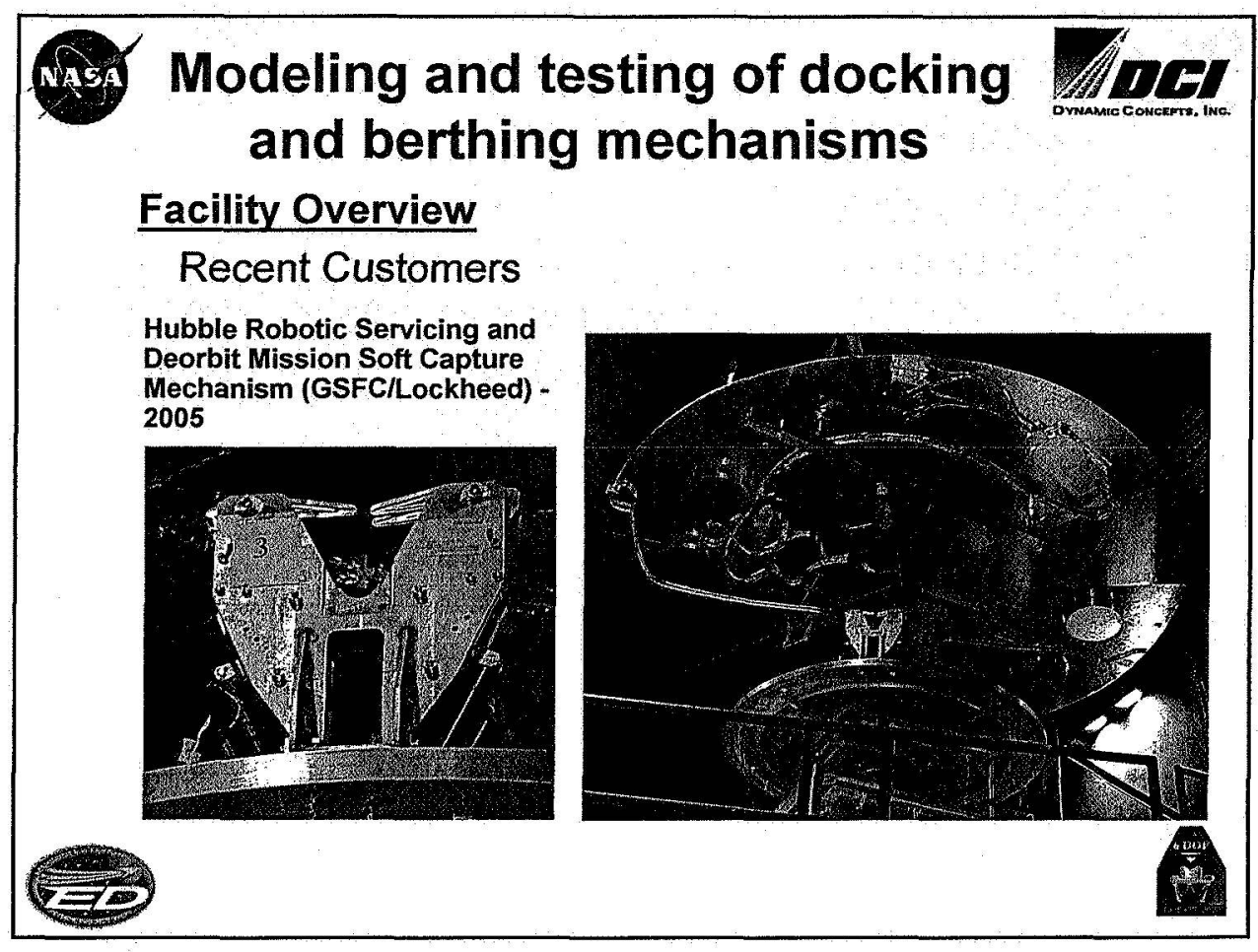

\section{Modeling and testing of docking} and berthing mechanisms

Modeling and Simulation

- Derive requirements from test objectives

- Develop analytical mechanism model

- Integrate mechanism model with two body simulation

- Monte Carlo analysis

- Determine CDSL component test requirements

- Determine test cases

- Correlate/update model with test results. 

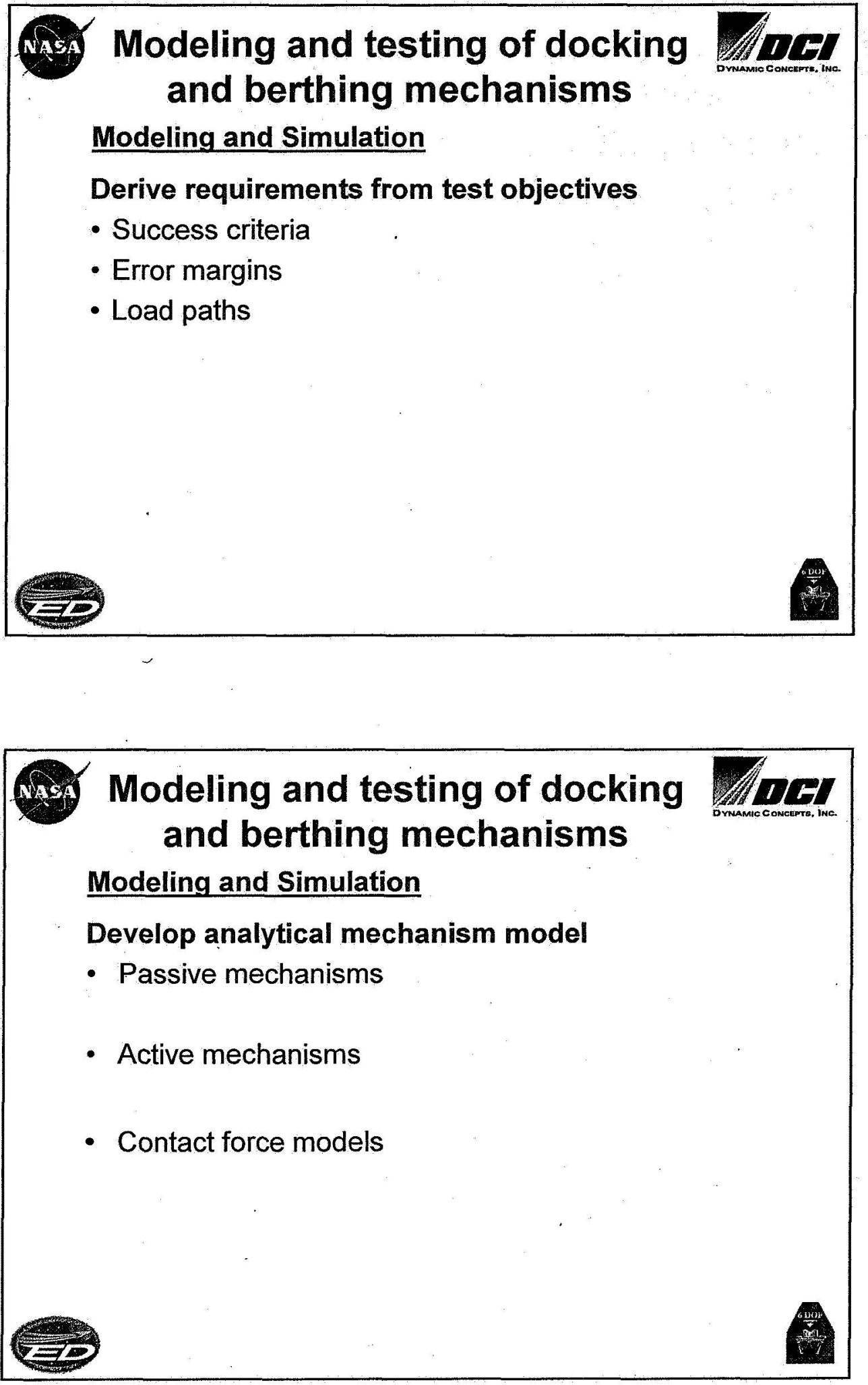


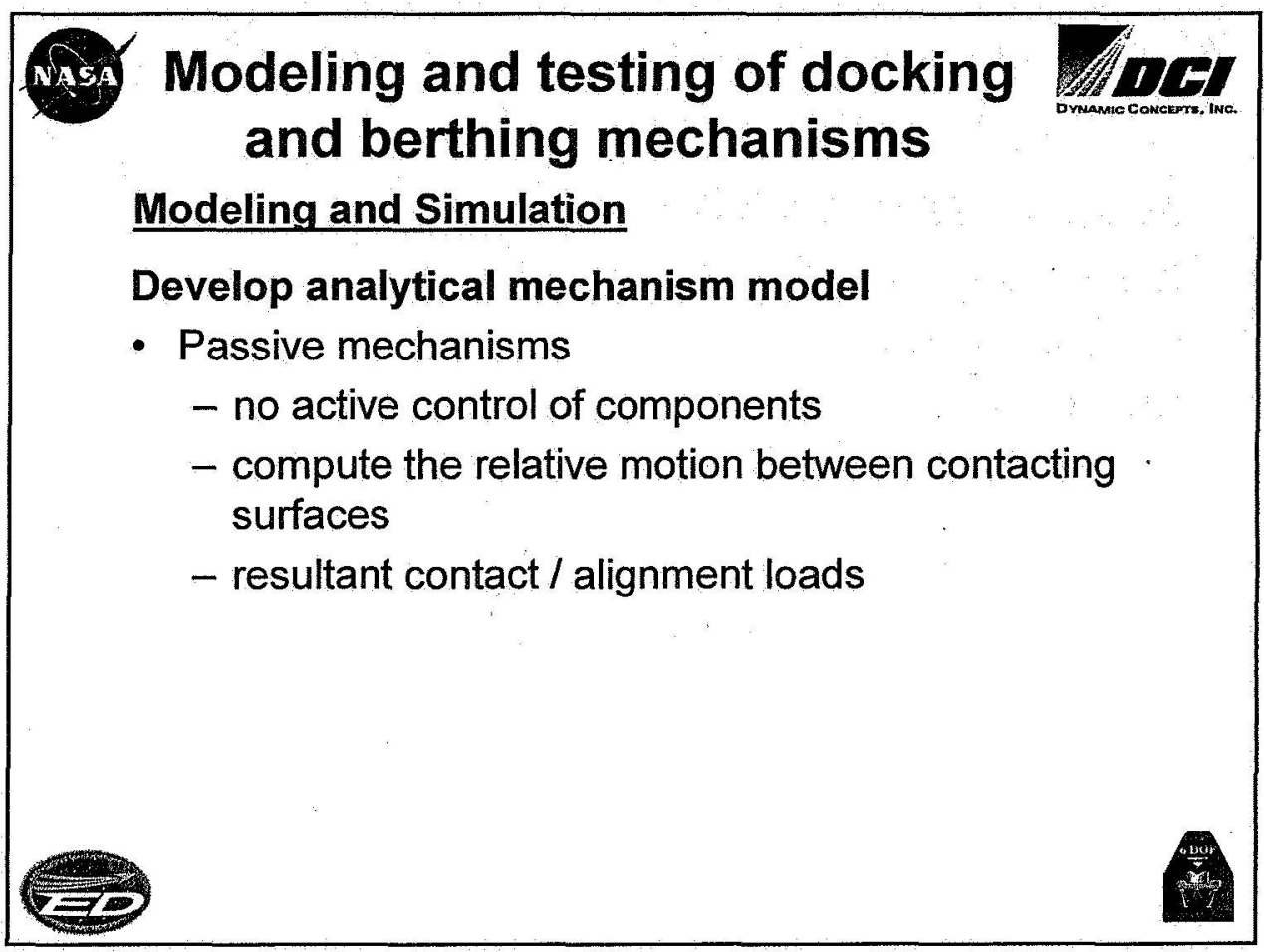

Modeling and testing of docking
and berthing mechanisms
Modeling and Simulation
Develop analytical mechanism model
- Active mechanisms
- usually more complex systems (Common Berthing
Mechanism)
- models also include modules for:
- mechanism sensors
- actuators
- control systems
- capture system dynamics 

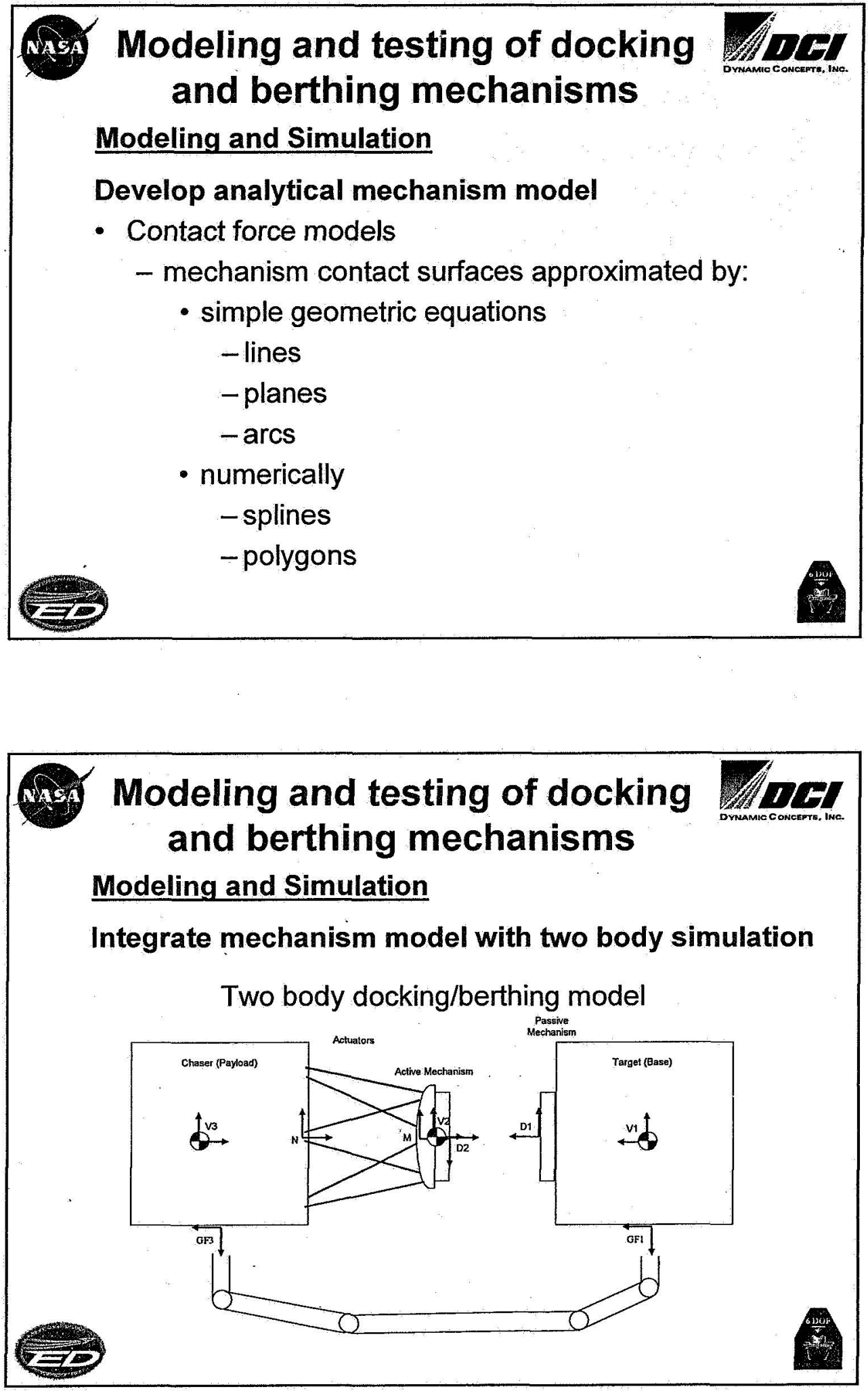


Modeling and testing of docking
and berthing mechanisms
Modeling and Simulation
Monte Carlo analysis
- Determine CDSL component requirements
- Screen Monte Carlo results for:
- Maximum loads
- Displacements
- Velocities
- Use these results to determine requirements for:
- Facility force-moment sensors
- Safety systems
- Test article instrumentation

\section{Nas Modeling and testing of docking and berthing mechanisms}

Modeling and Simulation

Monte Carlo analysis

- Determine test cases

- Generate random initial conditions

- Statistically reduce the non-contact initial conditions

- Define additional cases to isolate specific types of contact to support correlation with the analytical mechanism model

- Generate appropriate input files for the simulation 


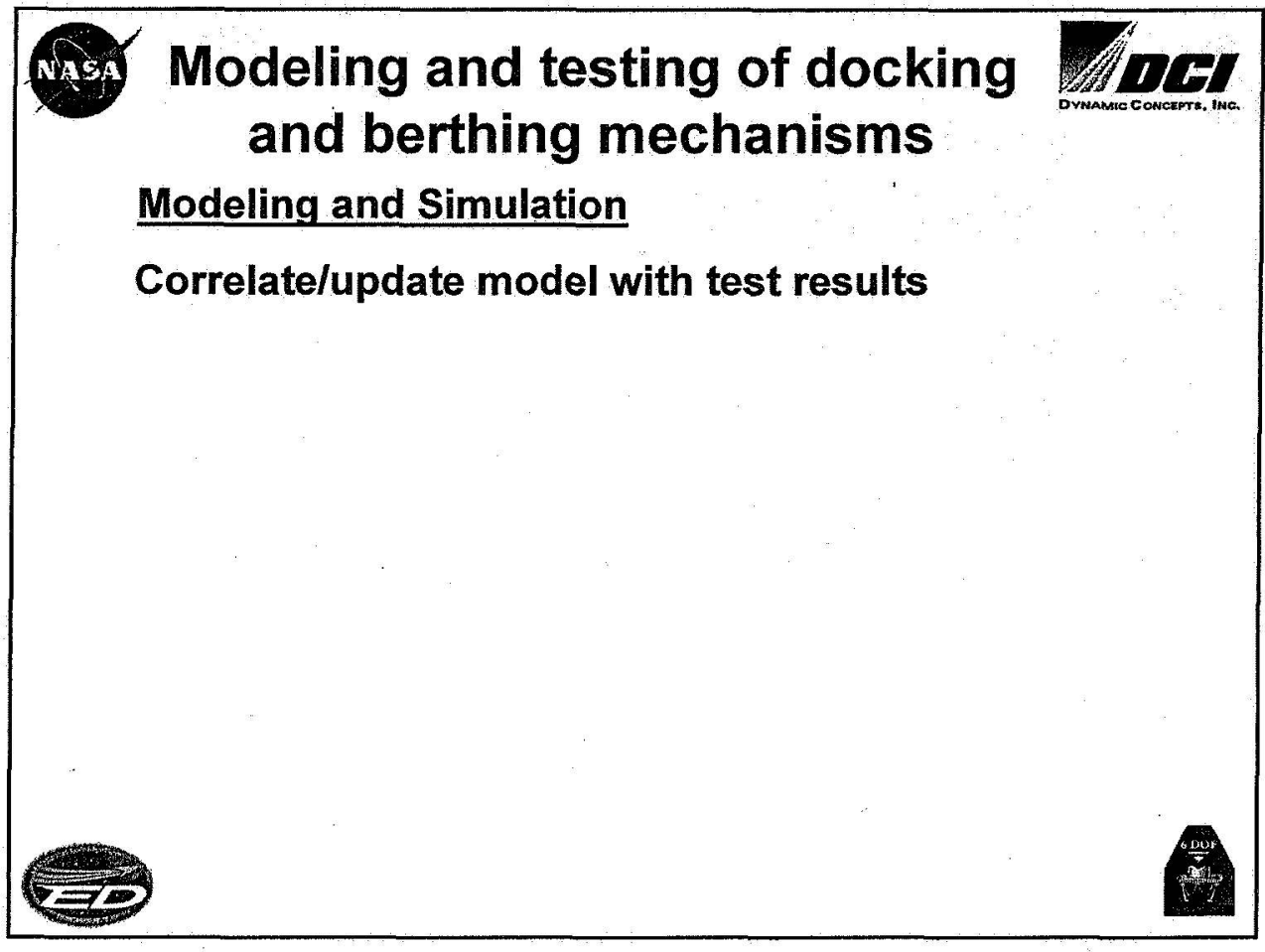

\section{(4as) Modeling and testing of docking and berthing mechanisms}

\section{Modeling and Simulation}

\section{Benefits}

- predict vehicle / manipulator interface loads

- predict mechanism component loads

- predict dynamic capture envelopes

- aid in the design of capture mechanism and alignment surfaces

- develop integrated operation procedures with vehicle and manipulator control systems

- define test fixture requirements 


\section{Modeling and testing of docking}

and berthing mechanisms

\section{Modeling and Simulation}

Uses

- pre-flight analysis

- procedure development

- training

- flight anomaly resolution

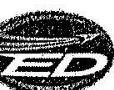

Mag. Modeling and testing of docking and berthing mechanisms

Modeling and Simulation

Recommendations

- Develop models early in the design cycle of the mating system to support the evaluation of design requirements and system performance

- Using results from prototype hardware and component/system testing, upgrade/update the mechanism models to correlate with test data facilitates the evaluation of future design improvements 


\section{Modeling and testing of docking and berthing mechanisms}

\section{Examples}

- Common Berthing Mechanism

- berthing mechanism that structurally connects components of the International Space Station

- Hubble Robotic Servicing and Deorbit Mission Soft Capture Mechanism

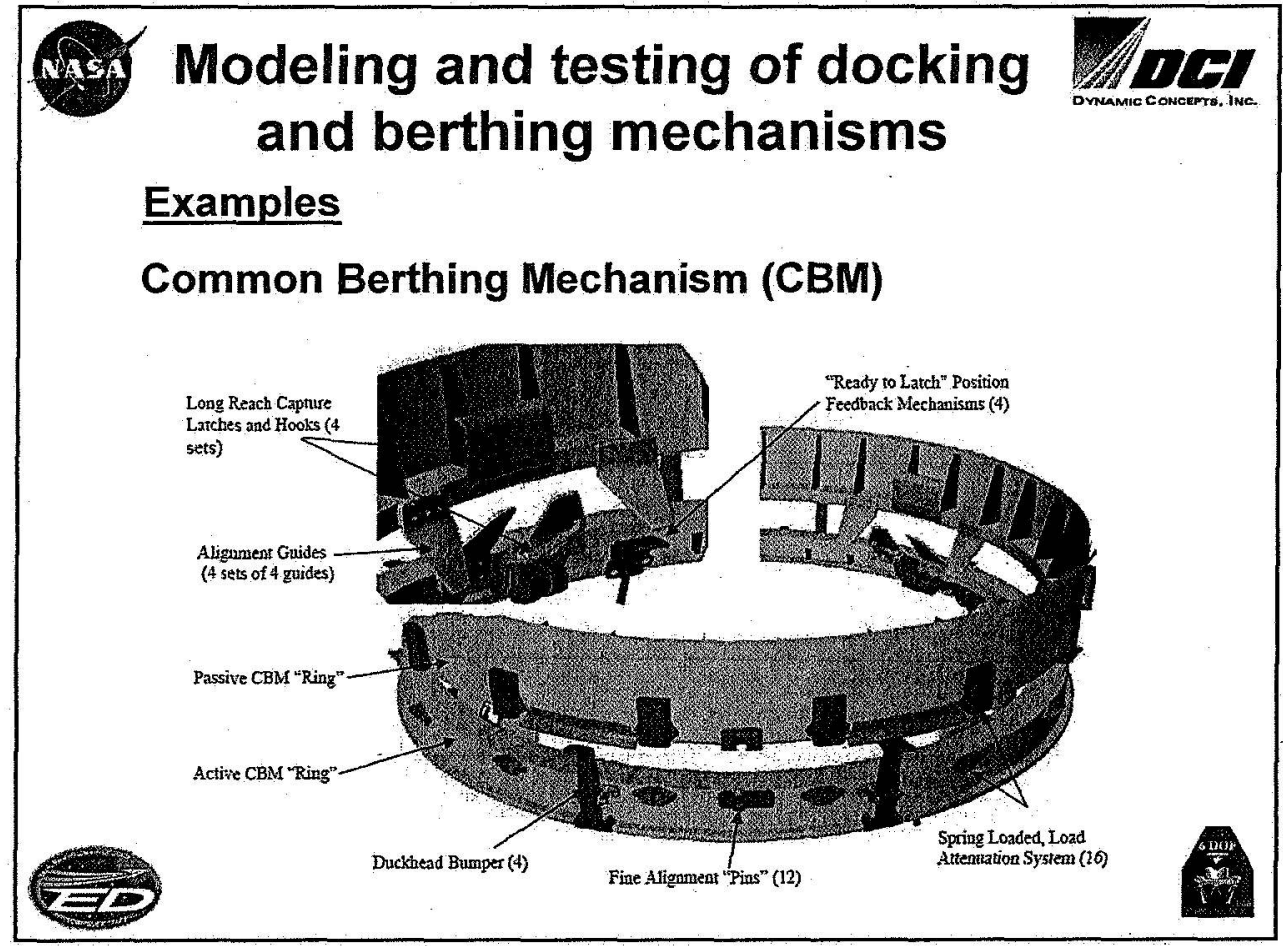




\section{Modeling and testing of docking and berthing mechanisms}

\section{Examples}

Common Berthing Mechanism
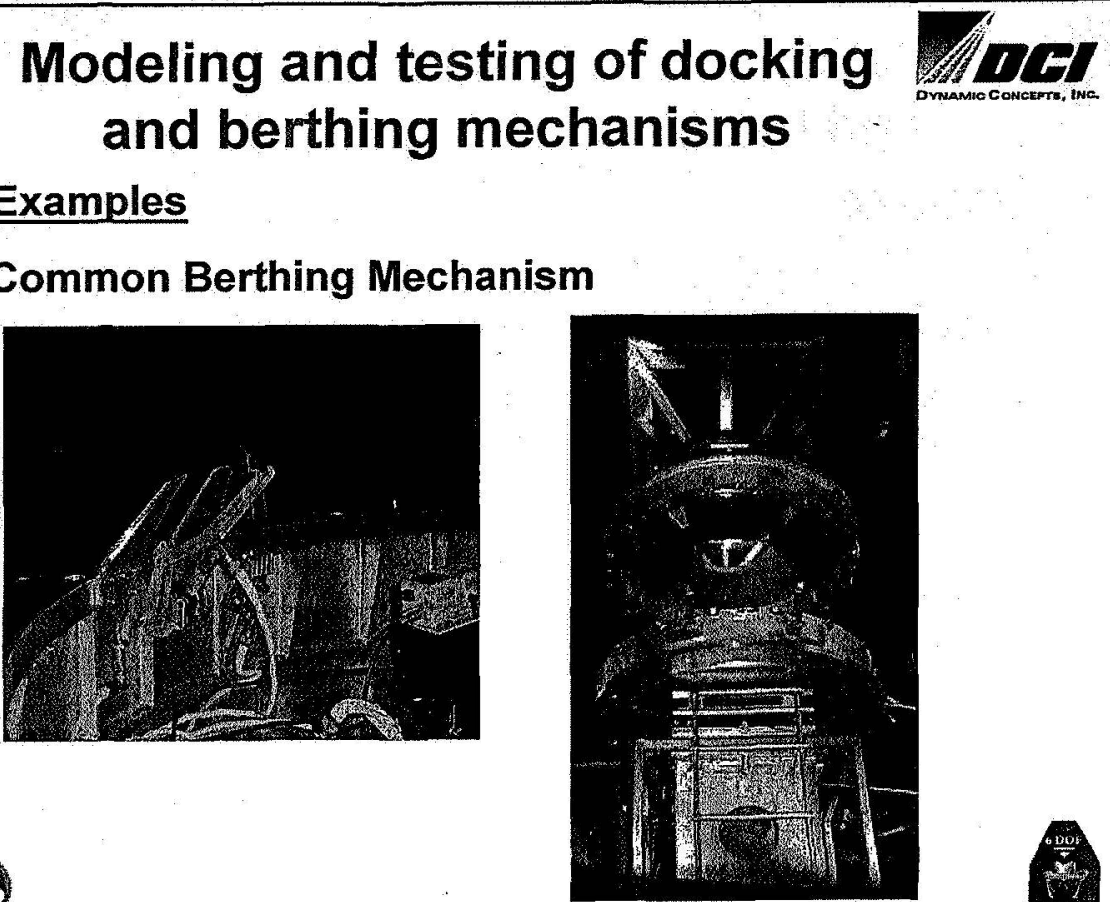

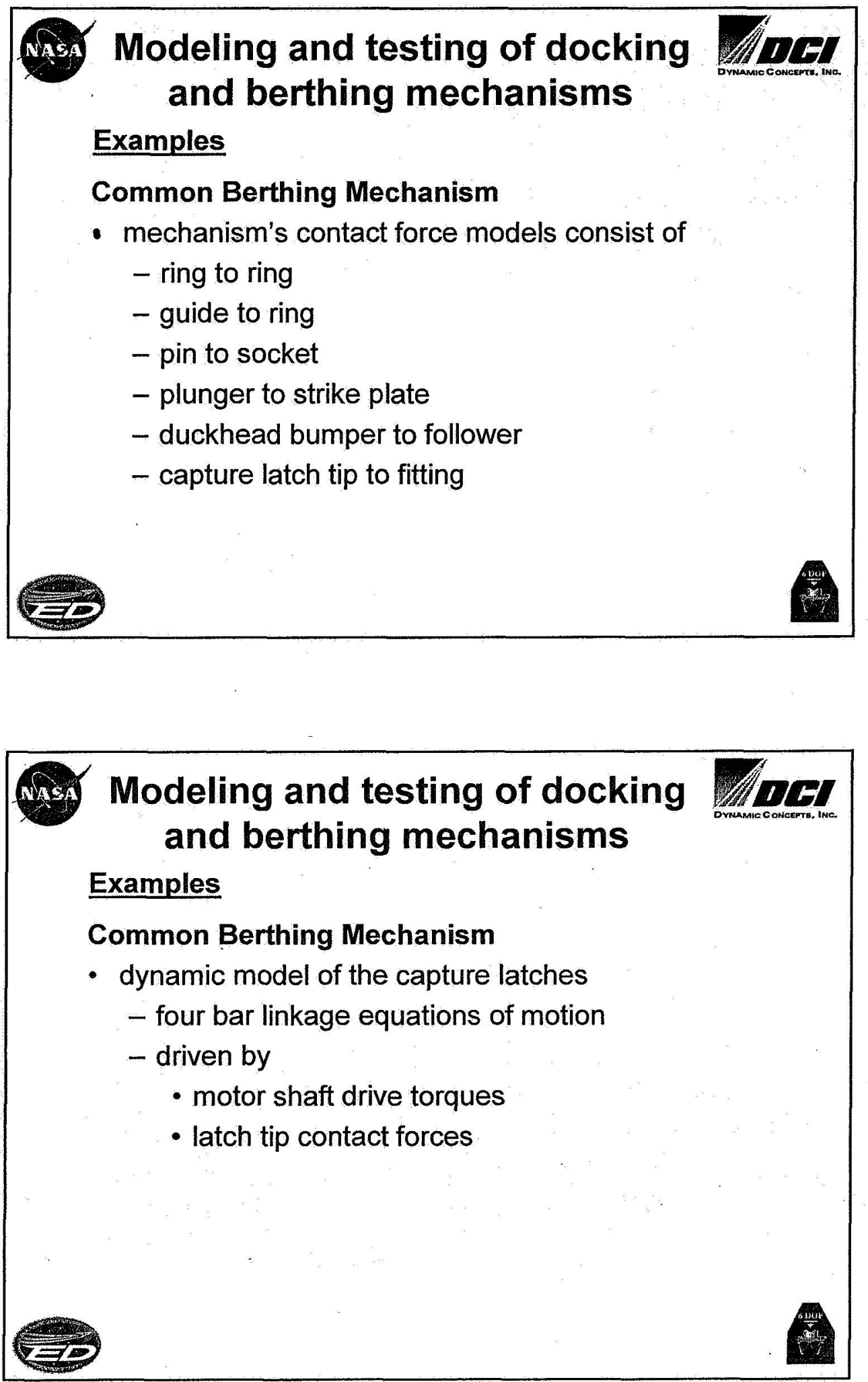


Modeling and testing of docking
and berthing mechanisms
Examples
Common Berthing Mechanism
- closed loop latch control system, drive train, motor, and
drive arm motion sensors
- simulated
- interfaced
- CBM operator
- capture latch dynamics

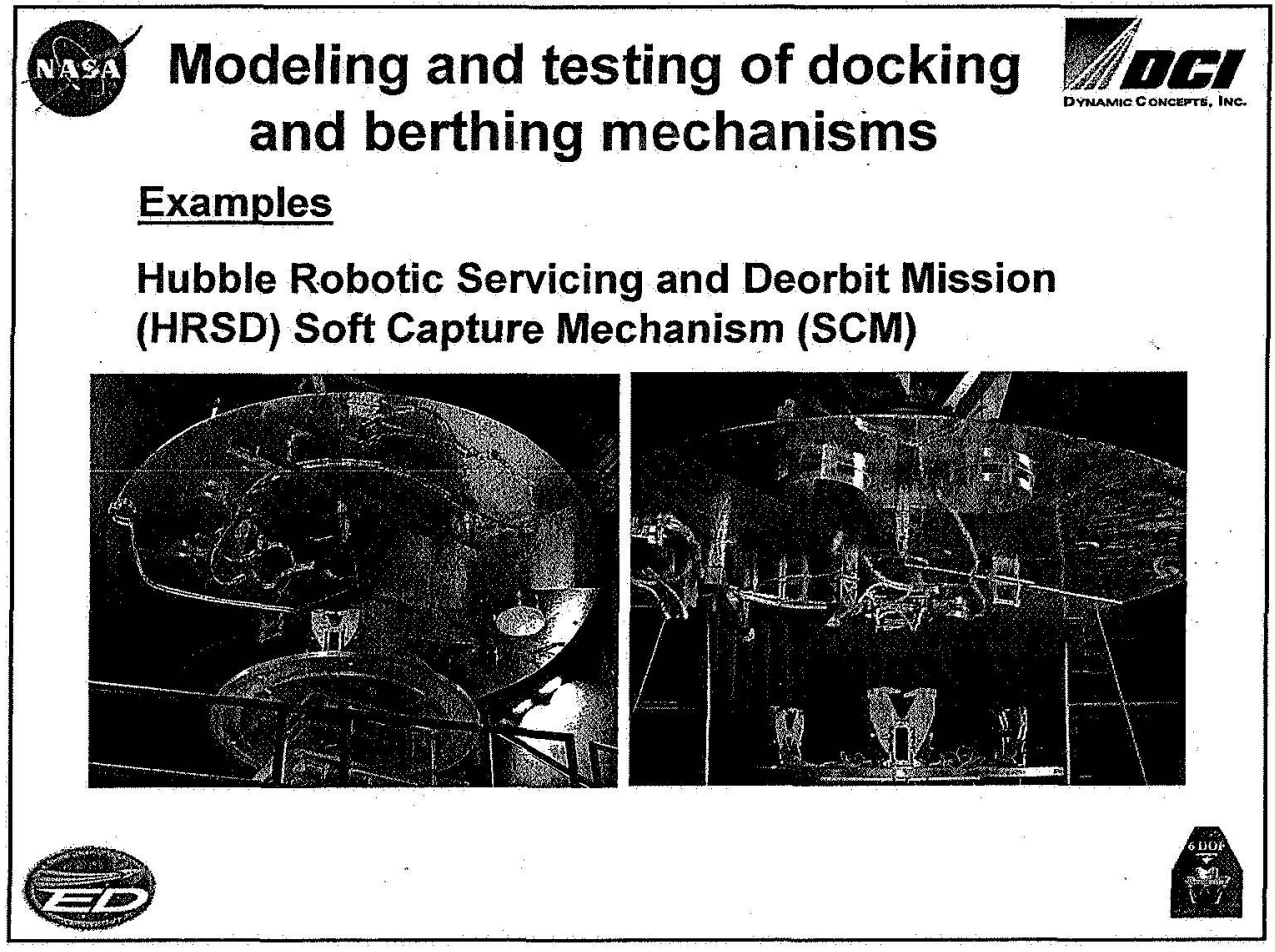




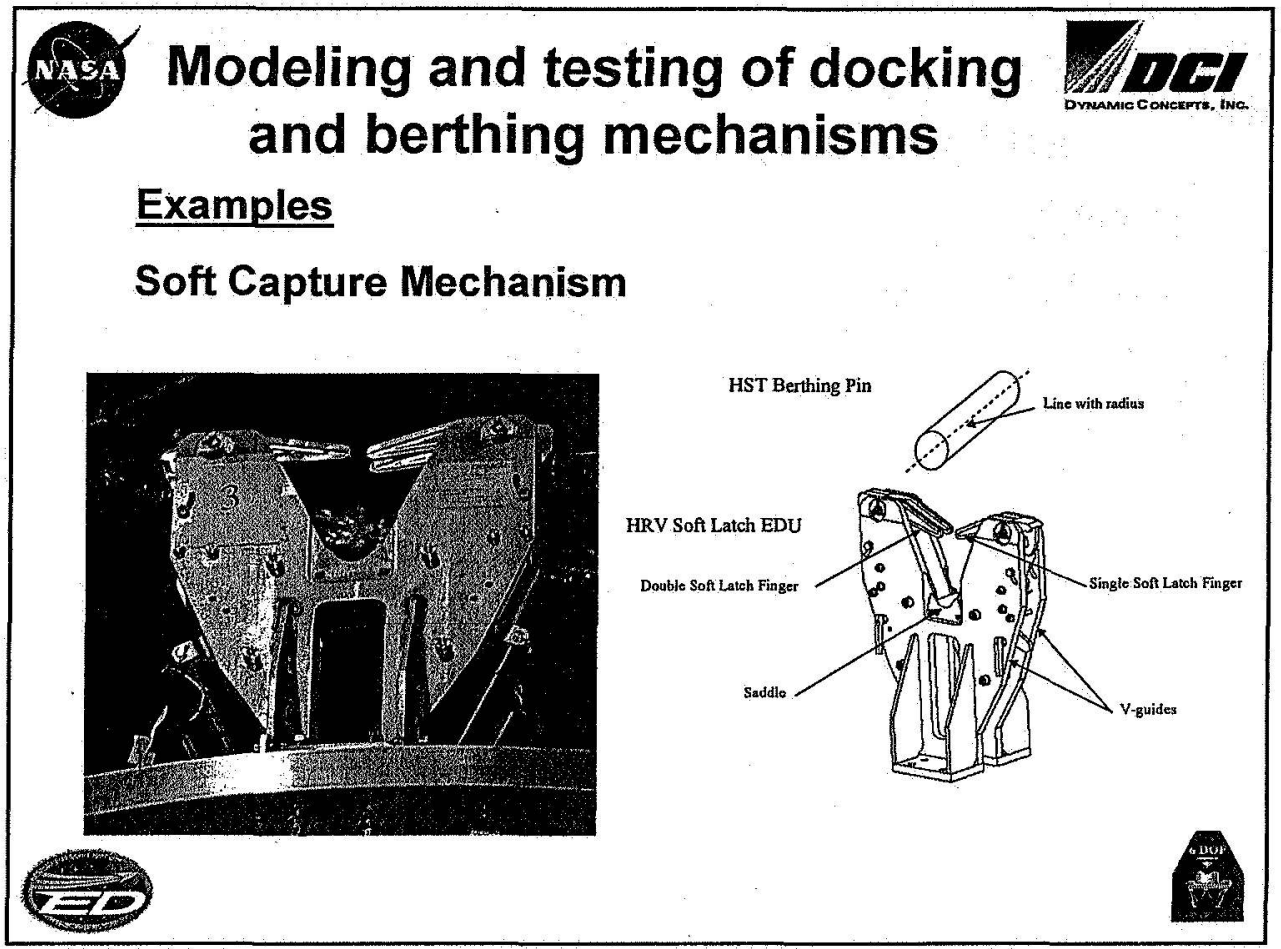

\section{(A) Modeling and testing of docking and berthing mechanisms}

\section{Examples}

\section{Soft Capture Mechanism}

- Mechanism contact force models

- SCM guide

- HST pin

- SCM saddle

- SCM soft capture fingers (single and double) 


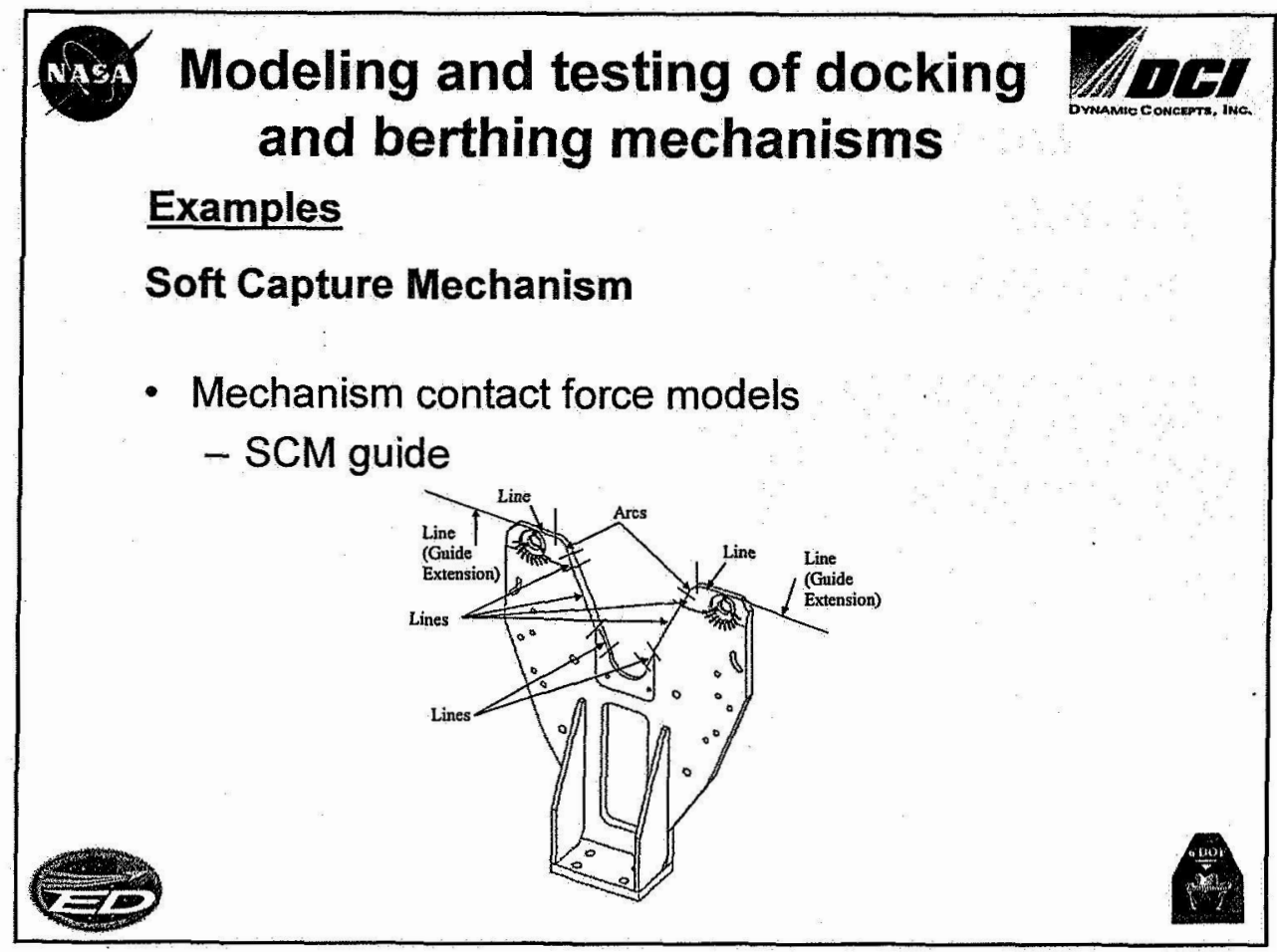

\section{(AS) Modeling and testing of docking and berthing mechanisms}

Examples

Soft Capture Mechanism

- Mechanism contact force models

- HST pin

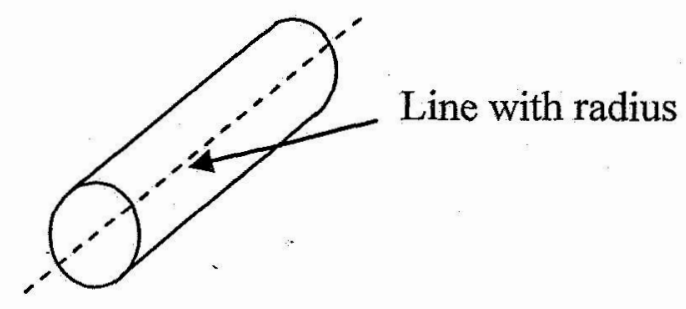




\section{Nas Modeling and testing of docking and berthing mechanisms}

\section{Examples}

Soft Capture Mechanism

- Mechanism contact force models

- SCM saddle

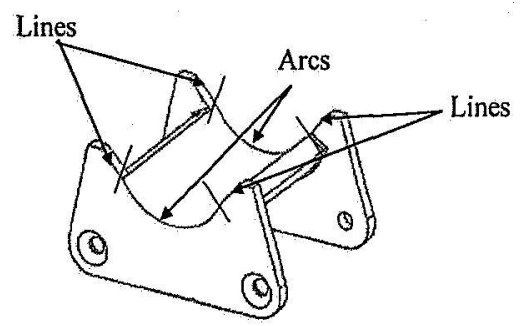

\section{MSA Modeling and testing of docking} and berthing mechanisms

\section{Examples}

\section{Soft Capture Mechanism}

- mechanism contact force models

- SCM soft capture fingers: single \& double (half)
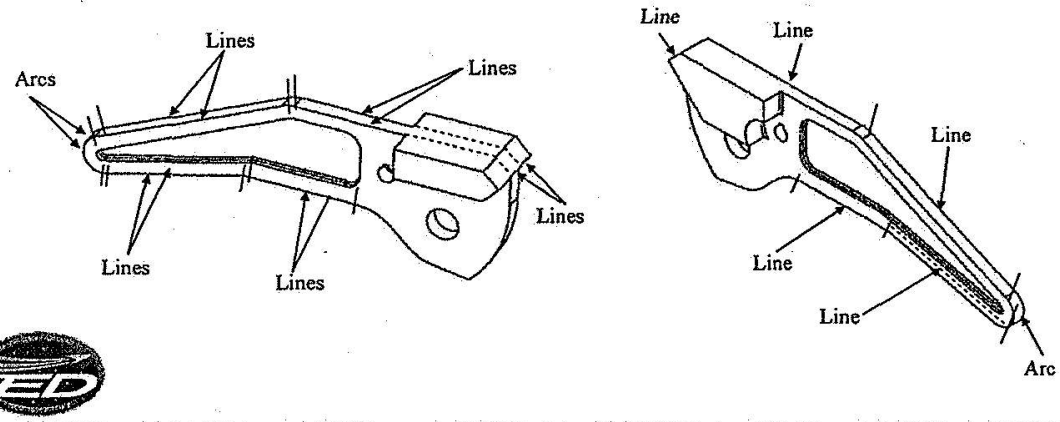

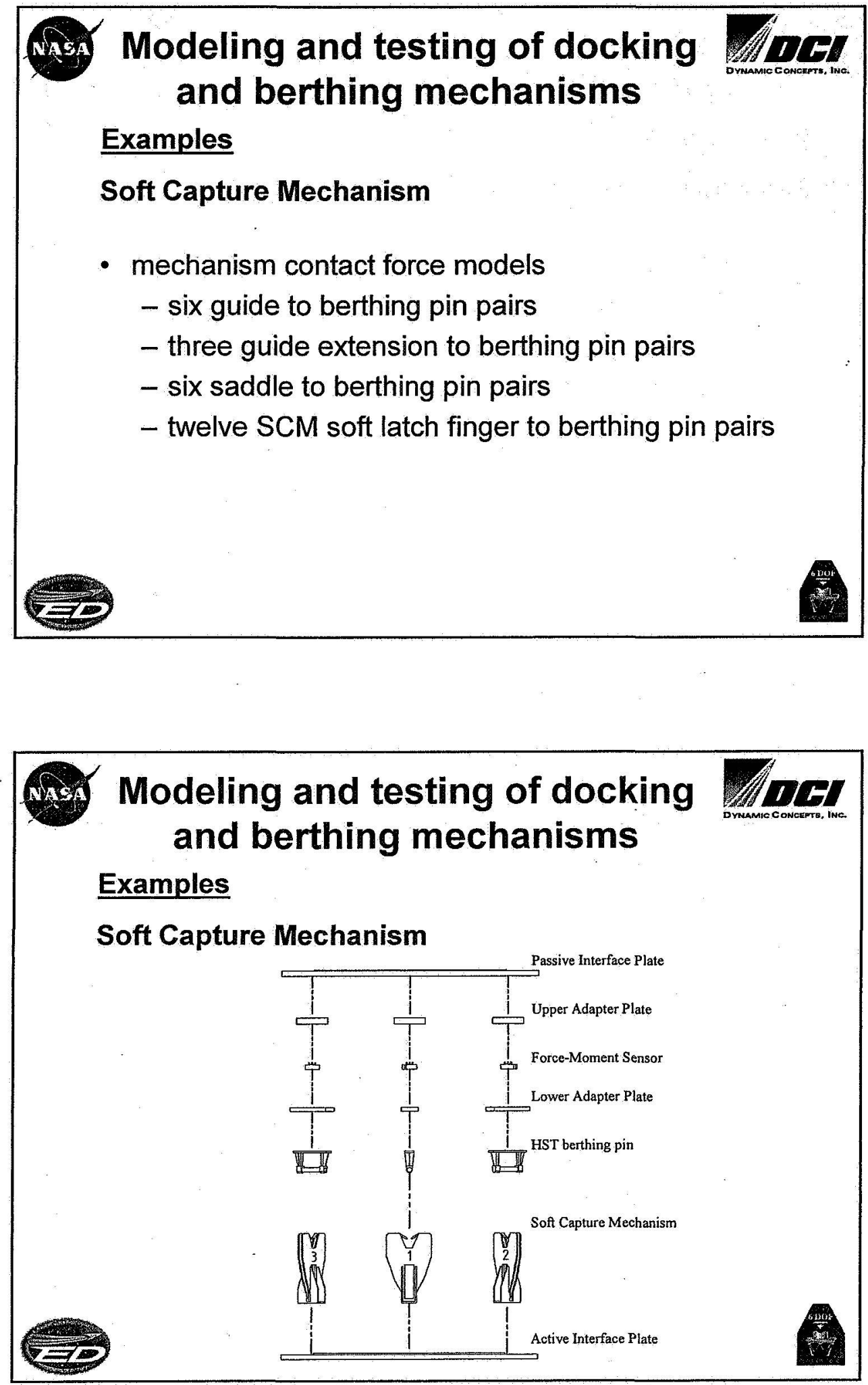


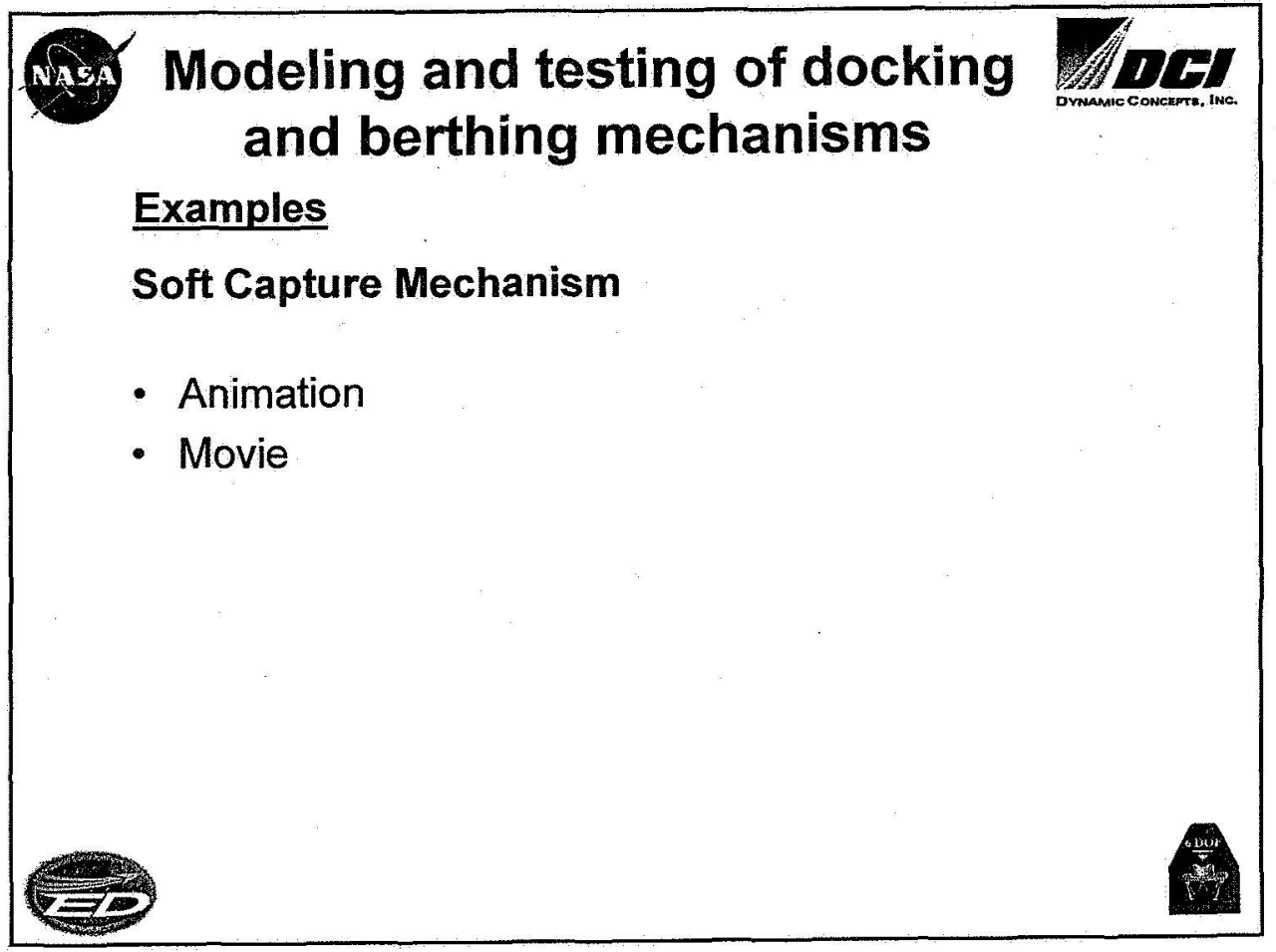

